\title{
ON THE USEFULNESS OF KNOWLEDGE OF ERROR VARIANCES IN THE CONSISTENT ESTIMATION OF AN UNREPLICATED ULTRASTRUCTURAL MODEL
}

\author{
SHALABH $^{\mathrm{a}, *}$, LEON J. GLESER ${ }^{\mathrm{b}, \dagger}$ and ORI ROSEN ${ }^{\mathrm{b}}$ \\ ${ }^{a}$ Department of Mathematics, Indian Institute of Technology, Kanpur 208016, India; \\ ${ }^{\mathrm{b}}$ Department of Statistics, University of Pittsburgh, Cathedral of Learning, \\ Pittsburgh, PA 15260, USA
}

(Received 1 September 2002; In final form 11 April 2003)

\begin{abstract}
This article considers an unreplicated ultrastructural model and discusses the asymptotic properties of three consistent estimators of slope parameter arising from the knowledge of measurement error variances. Conditions are deduced when knowing the error variances associated with both the study and the explanatory variables is more/less beneficial than using a single error variance in the formulation of slope estimators.
\end{abstract}

Keywords: Measurement errors; Reliability ratio; Error variance; Ultrastructural model

\section{INTRODUCTION}

One of the important requirements for the application of least squares procedure to a linear regression model is that the explanatory variables are measured without any error. Such a requirement may not be accomplished in many practical situations, and the available observations on one or more explanatory variables are subjected to measurement errors. Consequently, the optimal properties of the least squares procedure are vitiated, so much so that the estimators of the regression coefficients are not only biased but inconsistent too.

The quest for consistent estimators of the regression coefficients in the presence of measurement errors has led to the strategy of adjusting the least squares estimators when some additional information about the model is available; see, e.g., Cheng and Van Ness (1999) and Fuller (1987) for a comprehensive exposition. Among the various formulations of the additional information about the model parameters, there are three interesting specifications. In the context of a bivariate regression model, these relate to the specification of the variance of measurement errors associated with the study variable, explanatory variable or both in the form of their ratio. As all the three kinds of prior information allow us to estimate the parameters consistently, it is probably pertinent to find out which type of prior information is more useful in the sense of providing less biased and/or more efficient estimators.

\footnotetext{
* E-mail: shalabh1@yahoo.com

† Corresponding author. E-mail: ljg@stat.pitt.edu
} 
This issue is just touched upon by Cheng and Van Ness (1994, p. 180). This problem is addressed in this article and situations are identified where the utilization of both the error variances in the formulation of a consistent estimator of the slope parameter is worthwhile in comparison to the use of merely one error variance.

The exact (analytic) finite sample properties of the estimators based on the knowledge of one of the two measurement error variances are difficult to obtain, see, e.g., Cheng and Van Ness (1999, p. 57). In order to study the performance of these estimators, another alternative approach is to employ the large sample asymptotic approximation theory and base the inferences on these expressions. These expressions for efficiency properties involve some unknown population values and thus may leave the results unfit for practical applications. While dealing with measurement error models, it can be well appreciated that the reliability ratios associated with the study and explanatory variables are easily available or can be well estimated; see Gleser $(1992 ; 1993)$ for more details on this aspect. So under the given circumstances, an attempt is made in this paper to express the large sample asymptotic approximations for the three popular estimators in terms of reliability ratios associated with study and explanatory variables.

Further, the dominance conditions for the superiority of one estimator over the other are obtained in terms of reliability ratios. Their advantage is that in practical situations, generally one has the idea of reliability ratios of the dependent and independent variables. Based on this knowledge, one can decide and choose the estimator which will behave more efficiently.

The literature dealing with measurement error models generally assumes the measurement errors to have a normal distribution. The distribution of measurement errors essentially depends on the nature of the experiment involved and may not always be necessarily normal. Thus the specification of normality may lead to some incorrect and invalid statistical inferences. To study the impact of not necessarily normally distributed measurement errors on the efficiency properties of consistent estimators, some investigations are reported in this article.

In order to study the finite sample properties of consistent estimators and to see how well the large sample asymptotic approximations hold for small samples, a Monte-Carlo experiment study is also reported.

The plan of the presentation is as follows. Section 2 describes the unreplicated ultrastructural model with not necessarily normally distributed errors and presents three estimators arising from the knowledge of measurement error variances. In Section 3, the asymptotic properties of these three estimators are analyzed and the implications of their properties are discussed for examining the question of whether knowledge of one variance over the other is more beneficial when the aim is to estimate the slope parameter consistently. The details of a Monte-Carlo experiment are reported in Section 4. Finally, some remarks are offered in Section 5. The derivation of results is presented in the Appendix.

\section{MODEL AND THE ESTIMATION OF SLOPE PARAMETER}

Let us consider the following unreplicated ultrastructural model:

$$
\begin{aligned}
& y_{i}=Y_{i}+u_{i} \\
& x_{i}=X_{i}+v_{i} \\
& Y_{i}=\alpha+\beta X_{i} \quad(i=1,2, \ldots, n)
\end{aligned}
$$

where $y_{i}$ and $x_{i}$ refer to the observed values with true counterparts as $Y_{i}$ and $X_{i}$ of the study variable and explanatory variable respectively, $u_{i}$ and $v_{i}$ are the measurement errors 
associated with them, $\alpha$ is the intercept term and $\beta$ is the slope parameter. For our present discussion, the conventional disturbance term in the regression relationship (equation error) is assumed to be subsumed in the measurement error of the study variable. However, because information about an equation error variance generally cannot be obtained in advance of a regression study, the practicality of using the last two estimators that we discuss, particularly the last one, in contexts where an equation error may be present, has been questioned (See Cheng and Van Ness, 1999, pp. $80 \mathrm{ff}$ ).

We assume that $X_{1}, X_{2}, \ldots, X_{n}$ are random variables with means $m_{1}, m_{2}, \ldots, m_{n}$ but, variance $\sigma_{w}^{2}$. Thus we can write

$$
X_{i}=m_{i}+w_{i}
$$

where $w_{i}$ is a random variable with mean zero.

It is assumed that $u_{1}, u_{2}, \ldots, u_{n}$ are independently and identically distributed with mean 0 , variance $\sigma_{u}^{2}$ and kurtosis coefficient $\gamma_{2 u}$. Likewise, $v_{1}, v_{2}, \ldots, v_{n}$ are independently and identically distributed with mean 0 , variance $\sigma_{v}^{2}$ and kurtosis coefficient $\gamma_{2 v}$. Similarly, $w_{1}, w_{2}, \ldots, w_{n}$ are independently and identically distributed with mean 0 and variance $\sigma_{w}^{2}$. Further, all the three sets of random variables are mutually independent.

Combining (2.1)-(2.3), we can write

$$
y_{i}=\alpha+\beta x_{i}+\left(u_{i}-\beta v_{i}\right)
$$

Although this relationship mimics a regression model, application of the least squares procedure provides neither consistent nor unbiased estimators of the parameters. However, such estimators can be modified and consistency of the modified estimators can be achieved when some additional information related to the parameters of the model is available; see, e.g. Cheng and Van Ness (1999) and Fuller (1987).

When the variance $\sigma_{v}^{2}$ of the measurement errors associated with the explanatory variable is known, a consistent estimator of $\beta$ is given by

$$
b_{e}=\frac{s_{x y}}{s_{x x}-\sigma_{v}^{2}}
$$

where

$$
\begin{aligned}
& s_{x x}=\frac{1}{n} \sum_{1}^{n}\left(x_{i}-\bar{x}\right)^{2} ; \quad \bar{x}=\frac{1}{n} \sum_{1}^{n} x_{i} \\
& s_{x y}=\frac{1}{n} \sum_{1}^{n}\left(x_{i}-\bar{x}\right)\left(y_{i}-\bar{y}\right) ; \quad \bar{y}=\frac{1}{n} \sum_{1}^{n} y_{i} .
\end{aligned}
$$

When the variance $\sigma_{u}^{2}$ of the measurement errors associated with the study variable is known, a consistent estimator of $\beta$ is

$$
b_{s}=\frac{s_{y y}-\sigma_{u}^{2}}{s_{x y}},
$$

where

$$
s_{y y}=\frac{1}{n} \sum_{1}^{n}\left(y_{i}-\bar{y}\right)^{2} .
$$


Note The maximum likelihood estimator of $\beta$ when all random quantities in the model are normally distributed is equal to $b_{e}$ when $\sigma_{v}^{2}$ is known and $s_{x x}>\sigma_{v}^{2}$. Similarly, the maximum likelihood estimator of $\beta$ in normality contexts is equal to $b_{s}$ when $\sigma_{u}^{2}$ is known and $s_{y y}>\sigma_{u}^{2}$. However, here we are not necessarily assuming normality of all random quantities. Further, our primary interest is in the consistency and large-sample distributional properties of these estimators; thus, note that as $n$ goes to infinity, the probabilities of the events $\left\{s_{x x}>\sigma_{v}^{2}\right\}$ and $\left\{s_{y y}>\sigma_{u}^{2}\right\}$ converge to 1 .

In a similar manner, when both variances, or simply their ratio, is known, we can estimate $\beta$ by

$$
b_{e s}=t+\left(t^{2}+\frac{\sigma_{u}^{2}}{\sigma_{v}^{2}}\right)^{1 / 2}
$$

where

$$
t=\frac{1}{2 s_{x y}}\left(s_{y y}-\frac{\sigma_{u}^{2}}{\sigma_{v}^{2}} s_{x x}\right) ; \quad s_{x y} \neq 0 .
$$

The subscripts of $b$ indicate that estimators $b_{e}$ and $b_{s}$ arise from the knowledge of the error variances associated with the explanatory variable and study variable respectively while the estimator $b_{e s}$ is based on the knowledge of both error variances.

\section{ASYMPTOTIC PROPERTIES}

Asymptotic properties of the estimators (2.6) and (2.9) have been largely analyzed under the specification of normality of errors. For instance, Cheng and Van Ness (1991) have worked out the asymptotic distribution of $b_{e}$ along with the asymptotic properties of estimators of other parameters. Similar results for the estimators $b_{s}$ and $b_{e s}$ can be derived in the same way; see, e.g., Gleser (1985), Patefield (1978) and Wong (1993).

Relaxing the specification of normality of errors and assuming merely the finiteness of moments up to order four, Srivastava and Shalabh (1997a) have worked out asymptotic approximations for the bias and mean squared error of the estimator $b_{e}$; see also Srivastava and Shalabh (1997b). Following the same methodology, similar results for the estimators $b_{s}$ and $b_{e s}$ are derived in the Appendix and presented below.

In order to analyze the performance properties of estimators when errors are not necessarily normally distributed, we introduce the following notation:

$$
\begin{aligned}
s_{m m} & =\frac{1}{n} \sum_{1}^{n}\left(m_{i}-\bar{m}\right)^{2} ; \quad \bar{m}=\frac{1}{n} \sum_{1}^{n} m_{i} \\
\lambda_{x} & =\frac{s_{m m}+\sigma_{w}^{2}}{s_{m m}+\sigma_{w}^{2}+\sigma_{v}^{2}} ; \quad \lambda_{y}=\frac{\beta^{2}\left(s_{m m}+\sigma_{w}^{2}\right)}{\beta^{2}\left(s_{m m}+\sigma_{w}^{2}\right)+\sigma_{u}^{2}} \\
q & =\left(\frac{\lambda_{x}}{\lambda_{y}}\right)\left(\frac{1-\lambda_{y}}{1-\lambda_{x}}\right)
\end{aligned}
$$

and assume that $s_{m m}$ tends to a finite quantity as $n$ tends to infinity. Here, $\lambda_{x}$ and $\lambda_{y}$ are the reliability ratios of the explanatory variable and study variable respectively. 


\subsection{Comparison of Relative Biases}

Srivastava and Shalabh (1997a) have derived the large sample asymptotic approximation for the relative bias of the estimator $b_{e}$ to order $\mathrm{O}\left(n^{-1}\right)$ :

$$
\begin{aligned}
\operatorname{RB}\left(b_{e}\right) & =E\left(\frac{b_{e}-\beta}{\beta}\right) \\
& =\frac{\left(1-\lambda_{x}\right)}{n \lambda_{x}^{2}}\left[2+\lambda_{x}+\left(1-\lambda_{x}\right) \gamma_{2 v}\right]
\end{aligned}
$$

THEOREM 1 The large sample asymptotic approximations of the relative bias to order $\mathrm{O}\left(n^{-1}\right)$ for the estimators $b_{s}$ and $b_{e s}$ are given by

$$
\begin{aligned}
\operatorname{RB}\left(b_{s}\right) & =E\left(\frac{b_{s}-\beta}{\beta}\right) \\
& =\frac{\left(1-\lambda_{x}\right)}{n \lambda_{x}^{2}}\left[\lambda_{x}+q\left(1-3 \lambda_{x}\right)\right] \\
\operatorname{RB}\left(b_{e s}\right) & =E\left(\frac{b_{e s}-\beta}{\beta}\right) \\
& =\frac{\left(1-\lambda_{x}\right)}{n \lambda_{x}^{2}}\left[\frac{(2 q-1)\left(q+\lambda_{x}\right)}{q+1}+\frac{q^{3}\left(1-\lambda_{x}\right)}{(q+1)^{3}}\left(\gamma_{2 u}+\gamma_{2 v}\right)\right] .
\end{aligned}
$$

Proof See Appendix.

From the above results, it is interesting to note that asymmetry of the error distributions has no influence on the bias properties of the estimators, at least to the order of our approximation. However, the kurtosis does exhibit its influence in case of the estimators $b_{e}$ and $b_{e s}$.

Examining the expressions (3.1) and (3.2), we find that the relative bias to the given order of approximation in case of the estimator $b_{e}$ is positive as $\left(2+\gamma_{2 v}\right)$ cannot be negative. However, the relative bias of $b_{s}$ is positive as long as $\lambda_{x}$ is less than (1/3). This remains true for $\lambda_{x}$ greater than $(1 / 3)$ provided that

$$
q<\left(\frac{\lambda_{x}}{3 \lambda_{x}-1}\right)
$$

or equivalently

$$
\lambda_{x}<\left(\frac{1}{3-2 \lambda_{y}}\right) .
$$

When the condition (3.4) holds with a reversed inequality sign, the relative bias of $b_{s}$ is negative.

It is interesting to note that the bias of $b_{s}$ to the given order of approximation is the same for all kinds of error distribution. This is, however, not the case with $b_{e}$ and $b_{e s}$. Here the bias is influenced by the relative peakedness of the error distribution associated with the explanatory variable.

Let us now compare the estimators with respect to the criterion of the magnitude of bias. 
When the estimators $b_{e}$ and $b_{s}$ are biased in the same direction, $b_{s}$ has smaller magnitude of bias when any one of the following conditions holds true:

$$
\begin{aligned}
& q \leq\left[\frac{2+\left(1-\lambda_{x}\right) \gamma_{2 v}}{1-3 \lambda_{x}}\right] \quad \text { and } \lambda_{x}<\frac{1}{3} \\
& q \leq\left[\frac{\lambda_{x}}{3 \lambda_{x}-1}\right] \text { and } \lambda_{x}>\frac{1}{3}
\end{aligned}
$$

When the two estimators are biased in opposite directions, the condition for $b_{s}$ to have smaller magnitude of bias than $b_{e}$ is

$$
\left[\frac{\lambda_{x}}{3 \lambda_{x}-1}\right] \leq q \leq\left[\frac{2\left(1+\lambda_{x}\right)+\left(1-\lambda_{x}\right) \gamma_{2 v}}{3 \lambda_{x}-1}\right] \quad \text { and } \quad \lambda_{x}>\frac{1}{3}
$$

For $\lambda_{x}=1 / 3$, it is seen that the estimator $b_{s}$ has invariably smaller magnitude of bias than $b_{e}$, at least to the order of our approximation.

It may be observed that the condition (3.5) is satisfied for all kinds of error distributions so long as

$$
q \leq\left[\frac{2 \lambda_{x}}{1-3 \lambda_{x}}\right] \text { and } \quad \lambda_{x}<\frac{1}{3}
$$

where use has been made of the non-negativity of $\left(2+\gamma_{2 v}\right)$.

Similarly, the condition (3.7) holds true for all kinds of error distributions as long as

$$
\left[\frac{\lambda_{x}}{3 \lambda_{x}-1}\right] \leq q \leq\left[\frac{4 \lambda_{x}}{3 \lambda_{x}-1}\right] \quad \text { and } \quad \lambda_{x}>\frac{1}{3} .
$$

Utilizing these conditions, we have identified the cases for the superiority of $b_{s}$ over $b_{e}$ under the criterion of the magnitude of bias for a few selected values of the reliability ratios $\lambda_{x}$ and $\lambda_{y}$. These are assembled in Table I.

It is thus seen from Table I that the estimator $b_{s}$ possesses smaller magnitude of bias than $b_{s}$ over a fairly wide range of situations. In other words, for consistent estimation of the slope parameter, the use of $\sigma_{u}^{2}$ is a better proposition than the use of $\sigma_{v}^{2}$ unless $\lambda_{x}$ is sufficiently large.

TABLE I Statement of Cases Where $b_{s}$ has Smaller Magnitude of Bias than $b_{e}$.

\begin{tabular}{cccccccccc}
\hline \multicolumn{10}{c}{$\lambda_{x}$} \\
\cline { 2 - 9 } & 0.1 & 0.2 & 0.3 & 0.4 & 0.5 & 0.6 & 0.7 & 0.8 & 0.9 \\
\hline 0.1 & $\gamma_{2 v} \geq-1.4$ & $\gamma_{2 v} \geq-1.4$ & $*$ & $*$ & $\gamma_{2 v} \geq 3$ & $\gamma_{2 v} \geq 19$ & $\gamma_{2 v} \geq 65.7$ & $\gamma_{2 v} \geq 234$ & $\gamma_{2 v} \geq 1339$ \\
0.2 & $\gamma_{2 v} \geq-1.9$ & $*$ & $*$ & $*$ & $*$ & $\gamma_{2 v} \geq 4$ & $\gamma_{2 v} \geq 22.9$ & $\gamma_{2 v} \geq 94$ & $\gamma_{2 v} \geq 574$ \\
0.3 & $*$ & $*$ & $*$ & $*$ & $*$ & $\gamma_{2 v} \geq-1$ & $\gamma_{2 v} \geq 8.6$ & $\gamma_{2 v} \geq 47.3$ & $\gamma_{2 v} \geq 319$ \\
0.4 & $*$ & $*$ & $*$ & $*$ & $*$ & $*$ & $\gamma_{2 v} \geq 1.5$ & $\gamma_{2 v} \geq 24$ & $\gamma_{2 v} \geq 191.5$ \\
0.5 & $*$ & $*$ & $*$ & $*$ & $*$ & $*$ & $*$ & $\gamma_{2 v} \geq 10$ & $\gamma_{2 v} \geq 115$ \\
0.6 & $*$ & $*$ & $*$ & $*$ & $*$ & $*$ & $*$ & $\gamma_{2 v} \geq 0.7$ & $\gamma_{2 v} \geq 64$ \\
0.7 & $*$ & $*$ & $*$ & $*$ & $*$ & $*$ & $*$ & $*$ & $\gamma_{2 v} \geq 27.5$ \\
0.8 & $*$ & $*$ & $*$ & $*$ & $*$ & $*$ & $*$ & $*$ & $\gamma_{2 v} \geq 0.25$ \\
0.9 & $*$ & $*$ & $*$ & $*$ & $*$ & $*$ & $*$ & $*$ & $*$ \\
\hline
\end{tabular}


On the other hand, employing $\sigma_{v}^{2}$ in comparison to $\sigma_{u}^{2}$ in the formulation of a consistent estimator of $\beta$ under the criterion of magnitude of bias to the order of our approximation is better when

$$
\begin{aligned}
& q \geq\left[\frac{2+\left(1-\lambda_{x}\right) \gamma_{2 v}}{1-3 \lambda_{x}}\right] \quad \text { if } \lambda_{x}<\frac{1}{3} \\
& q \geq\left[\frac{2\left(1+\lambda_{x}\right)+\left(1-\lambda_{x}\right) \gamma_{2 v}}{3 \lambda_{x}-1}\right] \quad \text { if } \lambda_{x}>\frac{1}{3} .
\end{aligned}
$$

If we look at the expression (3.3) in order to compare the relative biases of $b_{e}$ and $b_{s}$ with $b_{e s}$, it is difficult to deduce any neat condition that can be given a simple and meaningful interpretation. It is therefore proposed to restrict our attention to the case of mesokurtic distributions $\left(\gamma_{2 u}=\gamma_{2 v}=0\right)$. Thus the expressions for the relative biases are

$$
\begin{aligned}
\operatorname{RB}\left(b_{e}\right)_{M} & =\frac{\left(1-\lambda_{x}\right)}{n \lambda_{x}^{2}}\left[2+\lambda_{x}\right] \\
\operatorname{RB}\left(b_{s}\right)_{M} & =\frac{\left(1-\lambda_{x}\right)}{n \lambda_{x}^{2}}\left[\lambda_{x}+q\left(1-3 \lambda_{x}\right)\right] \\
\operatorname{RB}\left(b_{e s}\right)_{M} & =\frac{\left(1-\lambda_{x}\right)}{n \lambda_{x}^{2}}\left[\frac{\left(q+\lambda_{x}\right)(2 q-1)}{q+1}\right] .
\end{aligned}
$$

It is thus seen that the relative biases of the estimators $b_{e}$ and $b_{e s}$ are always positive or negative depending upon whether or not the condition (3.4) is satisfied.

For the comparison of the magnitudes of the biases, we have computed the ratios

$$
\begin{aligned}
& r_{e}=\left|\frac{\operatorname{RB}\left(b_{e s}\right)_{M}}{\operatorname{RB}\left(b_{e}\right)_{M}}\right| \\
& r_{s}=\left|\frac{\operatorname{RB}\left(b_{e s}\right)_{M}}{\operatorname{RB}\left(b_{s}\right)_{M}}\right|
\end{aligned}
$$

for a few selected values of $\lambda_{x}$ and $\lambda_{y}$. These are assembled in Table II.

TABLE II Bias Ratios $r_{e}$ and $r_{s}$.

\begin{tabular}{cccccc}
\hline & \multicolumn{5}{c}{$\lambda_{y}$} \\
$\lambda_{x}$ & 0.1 & 0.3 & 0.5 & 0.7 & 0.9 \\
\cline { 2 - 6 } 0.1 & & & & & \\
$r_{e}$ & 0.262 & 0.065 & 0.070 & 0.061 & 0.052 \\
$r_{s}$ & 0.688 & 0.488 & 0.831 & 0.956 & 0.996 \\
0.3 & & & & & \\
$r_{e}$ & 2.498 & 0.283 & 0.032 & 0.112 & 0.131 \\
$r_{s}$ & 8.380 & 1.625 & 0.213 & 0.812 & 0.985 \\
0.5 & & & & & \\
$r_{e}$ & 6.460 & 1.246 & 0.300 & 0.037 & 0.171 \\
$r_{s}$ & 4.038 & 4.675 & $*$ & 0.325 & 0.963 \\
0.7 & & & & & \\
$r_{e}$ & 14.978 & 3.492 & 1.236 & 0.315 & 0.136 \\
$r_{s}$ & 1.805 & 1.783 & 1.788 & 2.125 & 0.884 \\
0.9 & & & & & \\
$r_{e}$ & 55.450 & 14.073 & 5.803 & 0.268 & 0.328 \\
$r_{s}$ & 1.176 & 1.173 & 1.169 & 1.162 & 1.188 \\
\hline
\end{tabular}

*The bias of $b_{s}$ vanishes and consequently the ratio $r_{s}$ is not available. 
As is observed from a comparison of (3.13) and (3.14), the relative bias of the estimator $b_{e s}$ is always smaller than that of $b_{e}$. Further, it is seen from Table II that the bias ratio $r_{e}$ for the estimators $b_{e s}$ and $b_{e}$ declines as $\lambda_{y}$ increases for a given value of $\lambda_{x}$ while it rises as $\lambda_{x}$ decreases for a given value of $\lambda_{y}$. So far as the estimators $b_{s}$ and $b_{e s}$ are concerned no clear pattern is found, but it is observed from Table II that the estimator $b_{s}$ outperforms $b_{e s}$ under the criterion of magnitude of bias over a wide range of values for the reliability ratios $\lambda_{x}$ and $\lambda_{y}$.

\subsection{Comparison of Relative Mean Squared Errors}

The relative mean squared error of the estimator $b_{e}$ to order $\mathrm{O}\left(n^{-1}\right)$ from Srivastava and Shalabh (1997a) is given by

$$
\begin{aligned}
\operatorname{RM}\left(b_{e}\right) & =E\left(\frac{b_{e}-\beta}{\beta}\right)^{2} \\
& =\frac{\left(1-\lambda_{x}\right)}{n \lambda_{x}^{2}}\left[\lambda_{x}+q+\left(1-\lambda_{x}\right)\left(2+\gamma_{2 v}\right)\right]
\end{aligned}
$$

Similar results for the estimators $b_{s}$ and $b_{e s}$ are presented below:

THEOREM 2 The large sample asymptotic approximations of the relative mean squared errors to order $\mathrm{O}\left(n^{-1}\right)$ for the estimators $b_{s}$ and $b_{e s}$ are given by

$$
\begin{aligned}
\operatorname{RM}\left(b_{s}\right) & =E\left(\frac{b_{s}-\beta}{\beta}\right)^{2} \\
& =\frac{\left(1-\lambda_{x}\right)}{n \lambda_{x}^{2}}\left[\lambda_{x}+q+q^{2}\left(1-\lambda_{x}\right)\left(2+\gamma_{2 u}\right)\right], \\
\operatorname{RM}\left(b_{e s}\right) & =E\left(\frac{b_{e s}-\beta}{\beta}\right)^{2} \\
& =\frac{\left(1-\lambda_{x}\right)}{n \lambda_{x}^{2}}\left[\lambda_{x}+q+\frac{q^{2}\left(1-\lambda_{x}\right)}{(q+1)^{2}}\left(\gamma_{2 u}+\gamma_{2 v}\right)\right] .
\end{aligned}
$$

As is observed in case of biases of the estimators, the mean squared errors are also influenced by the kurtosis, and not the skewness, of the distributions of measurement errors. Further, the mean squared errors are smaller when the error distributions are platykurtic rather than leptokurtic.

Comparing (3.15) and (3.16), we find that the estimator $b_{s}$ has smaller mean squared error than the estimator $b_{e}$ when

$$
q^{2}<\left(\frac{2+\gamma_{2 v}}{2+\gamma_{2 u}}\right) .
$$

The opposite is true, i.e., the estimator $b_{s}$ is less efficient than $b_{e}$ when the condition (3.18) holds with a reversed inequality sign.

Similarly, it is observed from (3.15) and (3.17) that the estimator $b_{e s}$ is more efficient than $b_{e}$ when

$$
\left(\frac{q+1}{q}\right)^{2}>\left(\frac{\gamma_{2 u}+\gamma_{2 v}}{2+\gamma_{2 v}}\right)
$$

which is always satisfied for all kinds of platykurtic distributions. 
When this inequality holds with an opposite sign, the estimator $b_{e s}$ is no more efficient than $b_{e}$.

Likewise, it follows from (3.16) and (3.17) that the estimator $b_{e s}$ has smaller mean squared error than $b_{s}$ when

$$
(q+1)^{2}>\left(\frac{\gamma_{2 u}+\gamma_{2 v}}{2+\gamma_{2 u}}\right)
$$

which is invariably true for platykurtic distributions.

When the condition (3.20) holds with a reversed inequality sign, the estimator $b_{s}$ is more efficient.

Now let us consider a particular case in which both the error distributions are mesokurtic $\left(\gamma_{2 u}=\gamma_{2 v}=0\right)$. In this case, the condition (3.18) reduces to the following

$$
q<-1
$$

or equivalently

$$
\lambda_{x}<\lambda_{y}
$$

while the conditions (3.19) and (3.20) always hold true. In other words, the estimator $b_{e s}$ based on the knowledge of the ratio of the error variances $\sigma_{u}^{2}$ and $\sigma_{v}^{2}$ is always more efficient than the estimators $b_{e}$ and $b_{s}$ based on the knowledge of a single error variance.

In order to appreciate the gain/loss in efficiency, we have evaluated the ratios

$$
\begin{aligned}
& \eta_{e}=\frac{\operatorname{RM}\left(b_{e}\right)}{\operatorname{RM}\left(b_{e s}\right)} \\
& \eta_{s}=\frac{\operatorname{RM}\left(b_{s}\right)}{\operatorname{RM}\left(b_{e s}\right)}
\end{aligned}
$$

for a few selected values of $\lambda_{x}$ and $\lambda_{y}$.

From Table III, it is seen that the efficiency ratio $\eta_{e}$ rises as $\lambda_{y}$ increases and/or $\lambda_{x}$ decreases. Just the reverse trend is observed in case of $\eta_{s}$, i.e., the efficiency ratio $\eta_{s}$ declines as $\lambda_{y}$ increases and/or $\lambda_{x}$ decreases. It is interesting to note that the gain in asymptotic efficiency of the estimator $b_{e s}$ over the estimators $b_{e}$ and $b_{s}$ is substantial for a fairly wide range of values of $\lambda_{x}$ and $\lambda_{y}$.

TABLE III Efficiency Ratios $\eta_{e}$ and $\eta_{s}$.

\begin{tabular}{cccccc}
\hline & \multicolumn{5}{c}{$\lambda_{y}$} \\
\cline { 2 - 6 }$\lambda_{x}$ & 0.1 & 0.3 & 0.5 & 0.7 & 0.9 \\
\hline 0.1 & & & & & \\
$\eta_{e}$ & 2.636 & 6.010 & 9.526 & 13.194 & 17.022 \\
$\eta_{s}$ & 2.636 & 1.337 & 1.105 & 1.027 & 1.002 \\
0.3 & & & & & \\
$\eta_{e}$ & 1.336 & 2.077 & 2.922 & 3.895 & 5.027 \\
$\eta_{s}$ & 6.010 & 2.077 & 1.353 & 1.098 & 1.009 \\
0.5 & & & & & \\
$\eta_{e}$ & 1.105 & 1.353 & 1.667 & 2.007 & 2.636 \\
$\eta_{s}$ & 9.526 & 2.922 & 1.667 & 1.198 & 1.020 \\
0.7 & & & & & \\
$\eta_{e}$ & 1.028 & 1.098 & 1.198 & 1.353 & 1.625 \\
$\eta_{s}$ & 13.194 & 3.895 & 2.077 & 1.353 & 1.042 \\
0.9 & & & & & \\
$\eta_{e}$ & 1.002 & 1.009 & 1.020 & 1.042 & 1.105 \\
$\eta_{s}$ & 17.022 & 5.027 & 2.636 & 1.625 & 1.105 \\
\hline
\end{tabular}




\section{MONTE-CARLO SIMULATION STUDY}

Before proceeding with the details of the Monte-Carlo simulation study, we may note that the exact finite sample properties of the two estimators, viz., $b_{e}$ and $b_{s}$ are difficult to obtain. The expected values of $b_{e}$ and $b_{s}$ do not exist. So the large sample asymptotic approximation theory is employed here to derive the first-order approximations for the bias and mean squared errors. The large sample asymptotic approximation theory essentially describes values in the central part of the distribution of the estimators; extreme values whose frequency becomes negligible in large samples are not described by the asymptotic theory. Even so, the large sample asymptotic approximation gives some idea about the overall behavior of the estimator. So the expressions for bias and mean squared errors derived earlier are not exact but at least explain the behavior of the estimator in the central part of the distribution.

In order to study the finite sample properties of the estimators $b_{e} b_{s}$ and $b_{e s}$, we conducted a Monte-Carlo simulation study using the S-plus statistical package. In order to study the behavior of non-normally distributed measurement errors, we adopted the following distributions:

(i) Central $t$-distribution (having no skewness but kurtosis only).

(ii) Beta distribution (having non-zero skewness and kurtosis) with density

$$
f(x)=\frac{1}{B(v, \omega)} x^{\nu-1}(1-x)^{\omega-1}, \quad 0 \leq x \leq 1,
$$

with $v=1.5$ and $\omega=3$, where $B(r, s)=\int_{0}^{1} u^{r-1}(1-u)^{s-1} \mathrm{~d} u$.

(iii) Weibull distribution (having non-zero skewness and kurtosis) with density

$$
f(x)=\left(\frac{b x^{b-1}}{a^{b}}\right) \exp \left[-\left(\frac{x}{a}\right)^{b}\right]
$$

with $a=1, b=0.5$.

(iv) Normal distribution (having no skewness and no kurtosis).

Note that because both $\sigma_{u}^{2}$ and $\sigma_{v}^{2}$ can take on values in $(0, \infty)$, there may be an infinite number of choices for their values. Instead, we base our choice on $\lambda_{x}$ and $\lambda_{y}$ which lie in $(0,1)$ and are easy to obtain or estimate in experimental settings; see, e.g., Gleser (1992) for details. Accordingly, we choose combinations of $\sigma_{u}^{2}, \sigma_{v}^{2}$ and $\sigma_{w}^{2}$ to obtain our specific choices for $\lambda_{x}$ and $\lambda_{y}$.

The following values are used in the simulation:

$$
\begin{aligned}
\lambda_{x} & =0.1,0.3,0.5,0.7,0.9, \\
\lambda_{y} & =0.1,0.3,0.5,0.7,0.9, \\
n & =15,43, \\
\beta & =0.3 .
\end{aligned}
$$

To provide values of the unknown means $m_{i}$, we used a data set from Moore (2000) for which $S_{m}^{2}=0.23$. Because our main interest focuses on the effect of non-normally distributed measurement errors, we let $w_{i}=\left(X_{i}-m_{i}\right)$ be normally distributed with mean 0 and suitably chosen values of $\sigma_{w}^{2}$ under different experimental settings.

The performance of the estimators in the simulation is presented in Tables IV-VII. All results are based on 5000 replications under conditions that make it possible to compute 
TABLE IV EVE and EMSE of Estimators When Measurement Errors Follow Normal Distribution.

\begin{tabular}{|c|c|c|c|c|c|c|c|c|c|c|c|c|c|}
\hline \multirow[b]{2}{*}{$\lambda_{x}$} & \multirow[b]{2}{*}{$\lambda_{y}$} & \multicolumn{2}{|c|}{$E V E\left(b_{e}\right)$} & \multicolumn{2}{|c|}{$E V E\left(b_{s}\right)$} & \multicolumn{2}{|c|}{$E V E\left(b_{e s}\right)$} & \multicolumn{2}{|c|}{$\operatorname{EMSE}\left(b_{e}\right)$} & \multicolumn{2}{|c|}{$E M S E\left(b_{s}\right)$} & \multicolumn{2}{|c|}{$E M S E\left(b_{e s}\right)$} \\
\hline & & $n=15$ & $n=43$ & $n=15$ & $n=43$ & $n=15$ & $n=43$ & $n=15$ & $n=43$ & $n=15$ & $n=43$ & $n=15$ & $n=43$ \\
\hline 0.1 & 0.1 & 0.396 & 0.347 & -0.001 & 0.185 & 1.028 & 1.047 & 19.205 & 9.949 & 8.463 & 11.040 & 14.973 & 18.556 \\
\hline 0.1 & 0.3 & 0.168 & 0.286 & 0.075 & 0.202 & 0.622 & 0.639 & 3.540 & 4.242 & 8.600 & 12.517 & 9.152 & 9.799 \\
\hline 0.1 & 0.5 & 0.172 & 0.352 & 0.196 & 0.350 & 0.605 & 0.542 & 3.539 & 3.252 & 15.893 & 7.505 & 10.093 & 5.472 \\
\hline 0.1 & 0.7 & 0.252 & 0.366 & 0.245 & 0.356 & 0.641 & 0.505 & 3.907 & 5.089 & 19.112 & 13.327 & 9.946 & 3.820 \\
\hline 0.1 & 0.9 & 0.217 & 0.372 & 0.291 & 0.355 & 0.556 & 0.476 & 2.940 & 4.936 & 10.464 & 8.349 & 3.727 & 1.785 \\
\hline 0.3 & 0.1 & 0.592 & 0.539 & 0.141 & 0.323 & 0.141 & 0.998 & 9.608 & 5.664 & 17.984 & 9.528 & 17.893 & 16.828 \\
\hline 0.3 & 0.3 & 0.609 & 0.667 & 0.274 & 0.335 & 0.583 & 0.440 & 4.468 & 10.271 & 7.590 & 6.468 & 6.947 & 1.190 \\
\hline 0.3 & 0.5 & 0.681 & 0.583 & 0.333 & 0.318 & 0.505 & 0.344 & 10.393 & 6.027 & 1.935 & 2.366 & 5.949 & 0.075 \\
\hline 0.3 & 0.7 & 0.691 & 0.583 & 0.318 & 0.321 & 0.352 & 0.324 & 10.486 & 4.729 & 0.548 & 0.011 & 0.070 & 0.015 \\
\hline 0.3 & 0.9 & 0.325 & 0.573 & 0.270 & 0.320 & 0.581 & 0.320 & 5.151 & 5.670 & 11.250 & 0.015 & 5.523 & 0.011 \\
\hline 0.5 & 0.1 & 0.566 & 0.344 & 0.309 & 0.330 & 2.128 & 1.125 & 8.095 & 0.087 & 8.060 & 8.018 & 49.870 & 27.768 \\
\hline 0.5 & 0.3 & 0.581 & 0.432 & 0.307 & 0.313 & 0.631 & 0.339 & 4.943 & 0.206 & 5.056 & 1.393 & 4.613 & 0.317 \\
\hline 0.5 & 0.5 & 0.645 & 0.333 & 0.314 & 0.301 & 0.396 & 0.307 & 8.068 & 0.015 & 0.576 & 0.007 & 1.529 & 0.004 \\
\hline 0.5 & 0.7 & 0.600 & 0.372 & 0.354 & 0.307 & 0.486 & 0.312 & 6.844 & 0.129 & 6.961 & 0.007 & 2.969 & 0.006 \\
\hline 0.5 & 0.9 & 0.673 & 0.319 & 0.324 & 0.304 & 0.339 & 0.304 & 9.563 & 0.005 & 0.058 & 0.006 & 0.094 & 0.001 \\
\hline 0.7 & 0.1 & 0.361 & 0.313 & 0.348 & 0.437 & 3.300 & 1.158 & 0.276 & 0.031 & 15.219 & 7.254 & 115.840 & 41.270 \\
\hline 0.7 & 0.3 & 0.361 & 0.309 & 0.275 & 0.299 & 0.651 & 0.305 & 0.112 & 0.008 & 2.323 & 0.025 & 12.157 & 0.007 \\
\hline 0.7 & 0.5 & 0.380 & 0.311 & 0.315 & 0.294 & 0.356 & 0.303 & 0.144 & 0.005 & 0.285 & 0.007 & 0.661 & 0.004 \\
\hline 0.7 & 0.7 & 0.324 & 0.317 & 0.295 & 0.299 & 0.308 & 0.306 & 0.008 & 0.005 & 0.005 & 0.004 & 0.004 & 0.003 \\
\hline 0.7 & 0.9 & 0.366 & 0.312 & 0.306 & 0.302 & 0.310 & 0.303 & 0.127 & 0.002 & 0.005 & 0.001 & 0.005 & 0.001 \\
\hline 0.9 & 0.1 & 0.307 & 0.306 & 0.319 & 0.449 & 4.940 & 0.962 & 0.090 & 0.026 & 15.924 & 4.920 & 251.142 & 46.639 \\
\hline 0.9 & 0.3 & 0.303 & 0.304 & 0.273 & 0.285 & 0.301 & 0.300 & 0.005 & 0.004 & 0.012 & 0.012 & 0.005 & 0.030 \\
\hline 0.9 & 0.5 & 0.299 & 0.301 & 0.274 & 0.292 & 0.301 & 0.300 & 0.005 & 0.002 & 0.015 & 0.003 & 0.005 & 0.001 \\
\hline 0.9 & 0.7 & 0.306 & 0.304 & 0.286 & 0.293 & 0.302 & 0.301 & 0.003 & 0.002 & 0.005 & 0.004 & 0.003 & 0.002 \\
\hline 0.9 & 0.9 & 0.306 & 0.305 & 0.299 & 0.299 & 0.302 & 0.302 & 0.002 & 0.001 & 0.001 & 0.001 & 0.001 & 0.001 \\
\hline
\end{tabular}


TABLE V EVE and EMSE of Estimators When Measurement Errors Follow $t$-Distribution.

\begin{tabular}{|c|c|c|c|c|c|c|c|c|c|c|c|c|c|}
\hline \multirow[b]{2}{*}{$\lambda_{x}$} & \multirow[b]{2}{*}{$\lambda_{y}$} & \multicolumn{2}{|c|}{$E V E\left(b_{e}\right)$} & \multicolumn{2}{|c|}{$E V E\left(b_{s}\right)$} & \multicolumn{2}{|c|}{$E V E\left(b_{e s}\right)$} & \multicolumn{2}{|c|}{ EMSE $\left(b_{e}\right)$} & \multicolumn{2}{|c|}{ EMSE $\left(b_{s}\right)$} & \multicolumn{2}{|c|}{$E M S E\left(b_{e s}\right)$} \\
\hline & & $n=15$ & $n=43$ & $n=15$ & $n=43$ & $n=15$ & $n=43$ & $n=15$ & $n=43$ & $n=15$ & $n=43$ & $n=15$ & $n=43$ \\
\hline 0.1 & 0.1 & 0.165 & 0.287 & 0.072 & 0.083 & 1.052 & 0.919 & 9.659 & 5.195 & 13.311 & 16.680 & 16.685 & 12.034 \\
\hline 0.1 & 0.3 & 0.140 & 0.360 & 0.054 & 0.258 & 0.592 & 0.622 & 2.763 & 4.699 & 10.319 & 9.062 & 5.287 & 10.198 \\
\hline 0.1 & 0.5 & 0.393 & 0.362 & 0.139 & 0.355 & 0.597 & 0.557 & 8.002 & 4.305 & 13.774 & 14.567 & 10.580 & 5.276 \\
\hline 0.1 & 0.7 & 0.199 & 0.378 & 0.154 & 0.322 & 0.609 & 0.518 & 3.196 & 5.360 & 17.162 & 7.749 & 9.379 & 3.987 \\
\hline 0.1 & 0.9 & 0.207 & 0.339 & 0.369 & 0.368 & 0.599 & 0.516 & 2.480 & 2.981 & 11.654 & 6.572 & 5.754 & 4.977 \\
\hline 0.3 & 0.1 & 0.466 & 0.579 & 0.156 & 0.292 & 1.779 & 1.159 & 11.179 & 10.879 & 17.984 & 11.743 & 37.085 & 21.965 \\
\hline 0.3 & 0.3 & 0.510 & 0.615 & 0.204 & 0.362 & 0.783 & 0.413 & 6.791 & 5.422 & 8.913 & 2.752 & 9.564 & 1.209 \\
\hline 0.3 & 0.5 & 0.491 & 0.621 & 0.283 & 0.321 & 0.581 & 0.344 & 6.300 & 5.638 & 8.038 & 0.347 & 6.626 & 0.148 \\
\hline 0.3 & 0.7 & 0.577 & 0.591 & 0.371 & 0.325 & 0.500 & 0.328 & 10.634 & 5.333 & 8.785 & 0.028 & 3.815 & 0.026 \\
\hline 0.3 & 0.9 & 0.539 & 0.637 & 0.348 & 0.319 & 0.475 & 0.321 & 4.388 & 8.183 & 2.380 & 0.009 & 2.050 & 0.012 \\
\hline 0.5 & 0.1 & 0.540 & 0.373 & 0.253 & 0.409 & 1.969 & 1.277 & 6.096 & 0.237 & 18.063 & 9.923 & 42.351 & 32.458 \\
\hline 0.5 & 0.3 & 0.571 & 0.379 & 0.311 & 0.304 & 0.539 & 0.315 & 4.619 & 0.184 & 4.249 & 0.019 & 3.507 & 0.009 \\
\hline 0.5 & 0.5 & 0.602 & 0.379 & 0.326 & 0.308 & 0.370 & 0.314 & 4.797 & 0.286 & 1.277 & 0.087 & 0.385 & 0.011 \\
\hline 0.5 & 0.7 & 0.563 & 0.390 & 0.332 & 0.305 & 0.363 & 0.311 & 4.053 & 1.226 & 0.544 & 0.007 & 0.706 & 0.006 \\
\hline 0.5 & 0.9 & 0.589 & 0.397 & 0.369 & 0.307 & 0.403 & 0.309 & 5.487 & 0.720 & 2.100 & 0.003 & 0.464 & 0.004 \\
\hline 0.7 & 0.1 & 0.589 & 0.314 & 0.291 & 0.446 & 2.089 & 1.222 & 6.553 & 0.033 & 15.565 & 5.935 & 53.441 & 42.226 \\
\hline 0.7 & 0.3 & 0.527 & 0.311 & 0.239 & 0.311 & 0.549 & 0.305 & 3.195 & 0.010 & 7.471 & 0.040 & 7.801 & 0.009 \\
\hline 0.7 & 0.5 & 0.543 & 0.315 & 0.343 & 0.313 & 0.346 & 0.304 & 3.599 & 0.011 & 1.992 & 0.048 & 0.083 & 0.008 \\
\hline 0.7 & 0.7 & 0.545 & 0.313 & 0.321 & 0.299 & 0.328 & 0.305 & 3.565 & 0.004 & 0.068 & 0.003 & 0.020 & 0.020 \\
\hline 0.7 & 0.9 & 0.529 & 0.314 & 0.326 & 0.302 & 2.459 & 0.303 & 3.555 & 0.003 & 15.202 & 0.001 & 64.195 & 0.142 \\
\hline 0.9 & 0.1 & 0.310 & 0.301 & 0.398 & 0.387 & 4.153 & 0.755 & 0.072 & 0.023 & 17.149 & 4.810 & 228.828 & 35.047 \\
\hline 0.9 & 0.3 & 0.309 & 0.302 & 0.291 & 0.299 & 0.664 & 0.302 & 0.019 & 0.006 & 1.678 & 0.027 & 18.105 & 0.006 \\
\hline 0.9 & 0.5 & 0.309 & 0.302 & 0.291 & 0.287 & 0.300 & 0.301 & 0.009 & 0.002 & 0.038 & 0.008 & 0.204 & 0.002 \\
\hline 0.9 & 0.7 & 0.309 & 0.301 & 0.283 & 0.296 & 0.303 & 0.301 & 0.005 & 0.001 & 0.009 & 0.002 & 0.004 & 0.001 \\
\hline 0.9 & 0.9 & 0.307 & 0.303 & 0.298 & 0.299 & 0.302 & 0.301 & 0.002 & 0.001 & 0.002 & 0.001 & 0.001 & 0.001 \\
\hline
\end{tabular}


TABLE VI EVE and EMSE of Estimators When Measurement Errors Follow Beta Distribution.

\begin{tabular}{|c|c|c|c|c|c|c|c|c|c|c|c|c|c|}
\hline \multirow[b]{2}{*}{$\lambda_{x}$} & \multirow[b]{2}{*}{$\lambda_{y}$} & \multicolumn{2}{|c|}{$E V E\left(b_{e}\right)$} & \multicolumn{2}{|c|}{$E V E\left(b_{s}\right)$} & \multicolumn{2}{|c|}{$E V E\left(b_{e s}\right)$} & \multicolumn{2}{|c|}{ EMSE $\left(b_{e}\right)$} & \multicolumn{2}{|c|}{ EMSE ( $\left.b_{s}\right)$} & \multicolumn{2}{|c|}{ EMSE $\left(b_{e s}\right)$} \\
\hline & & $n=15$ & $n=43$ & $n=15$ & $n=43$ & $n=15$ & $n=43$ & $n=15$ & $n=43$ & $n=15$ & $n=43$ & $n=15$ & $n=43$ \\
\hline 0.1 & 0.1 & 0.142 & 0.307 & 0.039 & 0.109 & 1.037 & 0.959 & 4.268 & 7.050 & 8.776 & 15.160 & 18.037 & 15.155 \\
\hline 0.1 & 0.3 & 0.180 & 0.303 & 0.037 & 0.198 & 0.689 & 0.561 & 2.859 & 4.310 & 5.818 & 12.621 & 13.912 & 5.001 \\
\hline 0.1 & 0.5 & 0.184 & 0.256 & 0.134 & 0.285 & 0.553 & 0.607 & 2.095 & 3.122 & 9.635 & 13.481 & 8.226 & 6.028 \\
\hline 0.1 & 0.7 & 0.177 & 0.364 & 0.271 & 0.323 & 0.552 & 0.527 & 1.035 & 4.401 & 20.048 & 10.730 & 6.012 & 3.800 \\
\hline 0.1 & 0.9 & 0.447 & 0.356 & 0.236 & 0.369 & 0.710 & 0.567 & 4.489 & 5.348 & 20.132 & 5.840 & 45.606 & 7.317 \\
\hline 0.3 & 0.1 & 0.469 & 0.497 & 0.173 & 0.270 & 1.676 & 1.092 & 6.573 & 2.965 & 12.991 & 15.633 & 29.395 & 20.489 \\
\hline 0.3 & 0.3 & 0.552 & 0.551 & 0.271 & 0.339 & 0.271 & 0.394 & 11.010 & 3.281 & 8.733 & 2.559 & 8.733 & 1.578 \\
\hline 0.3 & 0.5 & 0.526 & 0.564 & 0.294 & 0.326 & 0.712 & 0.338 & 7.534 & 4.039 & 11.329 & 0.236 & 6.504 & 0.051 \\
\hline 0.3 & 0.7 & 0.509 & 0.556 & 0.291 & 0.327 & 0.484 & 0.331 & 3.541 & 3.706 & 6.576 & 0.023 & 1.963 & 0.024 \\
\hline 0.3 & 0.9 & 0.573 & 0.532 & 0.369 & 0.320 & 0.473 & 0.321 & 7.887 & 2.367 & 2.675 & 0.011 & 0.687 & 0.010 \\
\hline 0.5 & 0.1 & 0.162 & 0.377 & 0.026 & 0.340 & 2.820 & 1.373 & 10.271 & 0.353 & 28.571 & 15.281 & 61.614 & 37.178 \\
\hline 0.5 & 0.3 & -0.015 & 0.371 & -0.096 & 0.311 & 1.705 & 0.360 & 6.447 & 0.441 & 21.373 & 0.387 & 30.703 & 2.387 \\
\hline 0.5 & 0.5 & 0.047 & 0.356 & -0.035 & 0.303 & 1.175 & 0.313 & 5.983 & 0.069 & 8.239 & 0.017 & 20.841 & 0.025 \\
\hline 0.5 & 0.7 & 0.045 & 0.475 & 0.001 & 0.304 & 0.859 & 0.311 & 4.166 & 0.641 & 10.305 & 0.006 & 14.136 & 0.006 \\
\hline 0.5 & 0.9 & 0.025 & 0.368 & 0.026 & 0.306 & 0.489 & 0.310 & 2.726 & 0.159 & 4.992 & 0.004 & 6.504 & 0.004 \\
\hline 0.7 & 0.1 & 0.028 & 0.311 & -0.048 & 0.314 & 4.130 & 0.721 & 32.762 & 0.023 & 50.234 & 1.643 & 97.430 & 22.604 \\
\hline 0.7 & 0.3 & 0.044 & 0.308 & 0.073 & 0.290 & 2.221 & 0.303 & 13.198 & 0.007 & 21.080 & 0.018 & 41.287 & 0.005 \\
\hline 0.7 & 0.5 & 0.094 & 0.310 & 0.042 & 0.294 & 1.607 & 0.304 & 9.294 & 0.004 & 15.238 & 0.005 & 26.735 & 0.003 \\
\hline 0.7 & 0.7 & 0.047 & 0.308 & 0.078 & 0.298 & 1.167 & 0.302 & 5.837 & 0.002 & 10.885 & 0.002 & 20.736 & 0.002 \\
\hline 0.7 & 0.9 & 0.083 & 0.309 & 0.100 & 0.301 & 0.579 & 0.302 & 6.615 & 0.002 & 7.269 & 0.001 & 5.079 & 0.001 \\
\hline 0.9 & 0.1 & 0.423 & 0.304 & 0.169 & 0.399 & 6.547 & 1.360 & 48.733 & 0.033 & 65.212 & 7.773 & 181.887 & 70.160 \\
\hline 0.9 & 0.3 & 0.365 & 0.303 & 0.101 & 0.281 & 4.019 & 0.300 & 25.938 & 0.004 & 42.189 & 0.011 & 102.433 & 0.003 \\
\hline 0.9 & 0.5 & 0.387 & 0.303 & 0.083 & 0.286 & 2.586 & 0.301 & 19.376 & 0.004 & 27.129 & 0.011 & 46.736 & 0.004 \\
\hline 0.9 & 0.7 & 0.372 & 0.304 & 0.009 & 0.291 & 1.883 & 0.302 & 16.436 & 0.002 & 15.763 & 0.003 & 39.042 & 0.002 \\
\hline 0.9 & 0.9 & 0.431 & 0.303 & 0.101 & 0.298 & 0.799 & 0.301 & 6.693 & 0.001 & 12.826 & 0.001 & 7.924 & 0.001 \\
\hline
\end{tabular}


TABLE VII EVE and EMSE of Estimators When Measurement Errors Follow Weibull Distribution.

\begin{tabular}{|c|c|c|c|c|c|c|c|c|c|c|c|c|c|}
\hline \multirow[b]{2}{*}{$\lambda_{x}$} & \multirow[b]{2}{*}{$\lambda_{y}$} & \multicolumn{2}{|c|}{$E V E\left(b_{e}\right)$} & \multicolumn{2}{|c|}{$E V E\left(b_{s}\right)$} & \multicolumn{2}{|c|}{$E V E\left(b_{e s}\right)$} & \multicolumn{2}{|c|}{$E M S E\left(b_{e}\right)$} & \multicolumn{2}{|c|}{$E M S E\left(b_{s}\right)$} & \multicolumn{2}{|c|}{$E M S E\left(b_{e s}\right)$} \\
\hline & & $n=15$ & $n=43$ & $n=15$ & $n=43$ & $n=15$ & $n=43$ & $n=15$ & $n=43$ & $n=15$ & $n=43$ & $n=15$ & $n=43$ \\
\hline 0.1 & 0.1 & -3.022 & -2.902 & -4.932 & -7.046 & 5.651 & 6.331 & 183.652 & 159.865 & 300.389 & 410.916 & 304.023 & 357.095 \\
\hline 0.1 & 0.3 & -2.659 & -2.576 & -4.609 & -6.651 & 5.275 & 3.151 & 164.180 & 150.150 & 278.581 & 375.554 & 257.369 & 343.860 \\
\hline 0.1 & 0.5 & -2.796 & -2.153 & -4.325 & -6.430 & 5.015 & 6.404 & 196.174 & 123.220 & 261.251 & 354.869 & 246.061 & 326.449 \\
\hline 0.1 & 0.7 & -2.539 & -1.968 & -4.491 & -6.339 & 4.749 & 6.689 & 150.867 & 108.668 & 263.564 & 337.629 & 231.406 & 332.449 \\
\hline 0.1 & 0.9 & -2.361 & -1.984 & -4.304 & -5.700 & 4.748 & 6.120 & 144.330 & 115.968 & 277.962 & 311.771 & 224.951 & 284.780 \\
\hline 0.3 & 0.1 & -3.138 & -3.009 & -4.702 & -7.573 & 5.102 & 6.174 & 303.313 & 162.361 & 284.111 & 430.750 & 255.478 & 370.886 \\
\hline 0.3 & 0.3 & -2.963 & -2.908 & -4.728 & -6.951 & 5.672 & 6.274 & 180.803 & 160.176 & 300.164 & 376.528 & 311.013 & 350.059 \\
\hline 0.3 & 0.5 & -2.978 & -2.549 & -4.757 & -6.431 & 5.758 & 6.804 & 198.987 & 126.873 & 277.110 & 354.941 & 308.289 & 365.676 \\
\hline 0.3 & 0.7 & -2.660 & -2.526 & -4.667 & -6.633 & 5.339 & 6.764 & 174.446 & 132.475 & 283.609 & 347.306 & 267.556 & 351.547 \\
\hline 0.3 & 0.9 & -2.647 & -2.016 & -3.930 & -6.373 & 4.980 & 6.623 & 163.715 & 103.885 & 233.953 & 355.318 & 252.148 & 334.833 \\
\hline 0.5 & 0.1 & -2.687 & -3.474 & -5.385 & -7.792 & 5.744 & 5.222 & 180.811 & 188.401 & 346.871 & 457.846 & 316.865 & 310.006 \\
\hline 0.5 & 0.3 & -3.204 & -3.001 & -4.776 & -6.486 & 5.745 & 6.248 & 202.099 & 172.650 & 291.760 & 365.437 & 290.455 & 360.203 \\
\hline 0.5 & 0.5 & -2.802 & -2.835 & -5.237 & -7.452 & 5.696 & 6.514 & 170.849 & 151.589 & 362.232 & 414.214 & 292.094 & 360.665 \\
\hline 0.5 & 0.7 & -2.852 & -2.606 & -4.505 & -6.892 & 5.647 & 6.710 & 187.430 & 142.398 & 296.195 & 374.128 & 300.591 & 358.834 \\
\hline 0.5 & 0.9 & -3.726 & -2.347 & -4.504 & -6.128 & 5.163 & 6.474 & 237.969 & 134.724 & 283.078 & 322.630 & 289.601 & 337.546 \\
\hline 0.7 & 0.1 & -3.538 & -3.482 & -5.263 & -8.370 & 4.696 & 4.310 & 203.336 & 193.469 & 341.299 & 490.049 & 263.665 & 263.832 \\
\hline 0.7 & 0.3 & -3.059 & -3.223 & -4.918 & -7.882 & 4.758 & 4.152 & 203.409 & 181.598 & 293.312 & 460.293 & 248.521 & 246.752 \\
\hline 0.7 & 0.5 & -3.238 & -3.480 & -5.141 & -8.917 & 5.396 & 4.296 & 208.829 & 201.186 & 319.002 & 503.282 & 280.135 & 264.960 \\
\hline 0.7 & 0.7 & -3.228 & -2.675 & -5.256 & -6.907 & 5.578 & 6.280 & 213.734 & 147.219 & 326.517 & 375.156 & 287.208 & 352.006 \\
\hline 0.7 & 0.9 & -2.748 & -2.353 & -4.603 & -6.287 & 5.435 & 6.694 & 147.790 & 137.451 & 238.552 & 367.987 & 282.838 & 347.749 \\
\hline 0.9 & 0.1 & -2.748 & -3.975 & -4.603 & -9.441 & 5.435 & 2.897 & 147.790 & 222.964 & 288.552 & 526.850 & 282.838 & 185.490 \\
\hline 0.9 & 0.3 & -4.170 & -3.741 & -5.445 & -8.123 & 3.713 & 4.040 & 244.683 & 209.302 & 339.514 & 461.436 & 210.235 & 243.869 \\
\hline 0.9 & 0.5 & -3.140 & -3.376 & -5.201 & -8.063 & 4.680 & 4.665 & 200.619 & 177.249 & 327.270 & 442.289 & 252.164 & 285.720 \\
\hline 0.9 & 0.7 & -2.983 & -3.141 & -4.751 & -7.275 & 5.844 & 5.639 & 199.350 & 167.677 & 299.138 & 395.537 & 324.185 & 329.619 \\
\hline 0.9 & 0.9 & -2.596 & -2.906 & -4.902 & -7.378 & 5.677 & 6.267 & 181.793 & 162.119 & 328.501 & 419.617 & 300.202 & 345.011 \\
\hline
\end{tabular}


the estimates, viz., $s_{x x}>\sigma_{v}^{2}$ for $b_{e}, s_{y y}>\sigma_{u}^{2}$ for $b_{s}$ and $s_{x y} \neq 0$ for $b_{e s}$. The expected values of the estimators (EVE) and their expected mean squared errors (EMSE) are plotted in a 3-dimensional surface spline plot.

The overall relative performance of these estimators is best seen by comparing the surface spline plots of EVE and EMSE under different distributions with those under the normal distribution. Before presenting the results, we note again that if two surface spline plots under small and large samples for a particular distribution are quite similar, this suggests that the behavior of the estimators is explained satisfactorily by the large sample asymptotic results. The surface spline plots are constructed by putting the values of $\lambda_{x}$ on the $x$-axis (denoted as lambda_x), values of $\lambda_{y}$ on the $y$-axis (denoted as lambda_y) and values of EVEs and EMSEs of the three estimators $b_{e}$ (denoted as be), $b_{s}$ (denoted as bs) and
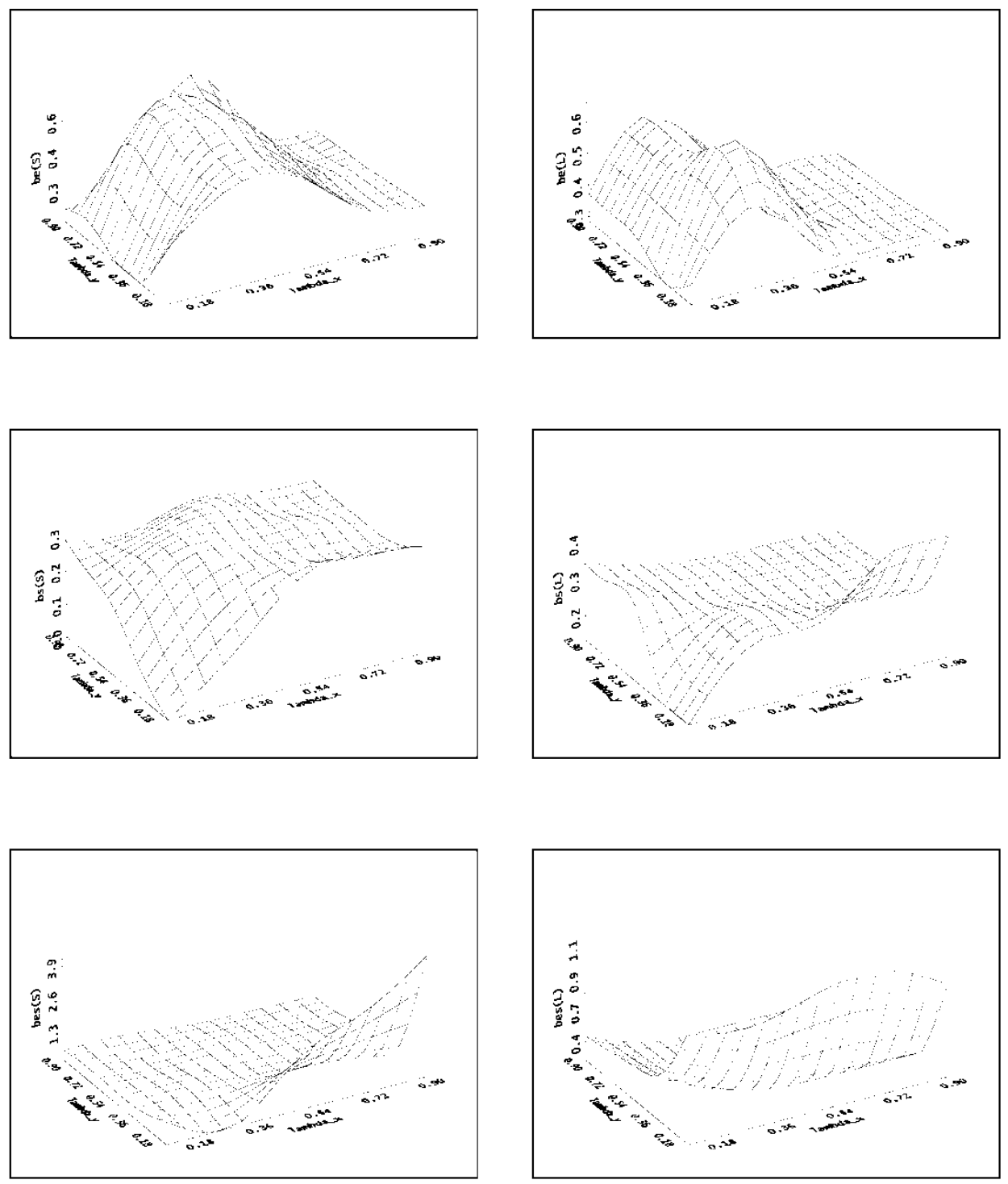

FIGURE 1 EVE with Normal distribution under small (S) and large (L) samples. The values on vertical axis are the EVEs of $b_{e}$ (be), $b_{s}$ (bs) and $b_{e s}$ (bes). 
$b_{e s}$ (denoted as bes) on the $z$-axis. The values under small and large samples are denoted by ' $\mathrm{S}$ ' and 'L' respectively.

Firstly, we compare the surface spline plots of the respective distributions under small samples $(n=15)$ and large samples $(n=43)$. The surface spline plots of EVE and EMSE of all the three estimators are quite similar in case of normal (Figs. 1 and 2) and $t$-distributed (Figs. 3 and 4) measurement errors except at few points, viz., when $\lambda_{x} \geq 0.5$ and $\lambda_{y} \geq 0.5$ in case of EVE and EMSE of $b_{e}$; when $\lambda_{x}=0.5$ and $\lambda_{y}=0.9$ in case of EVE of $b_{e s}$ and EMSE of $b_{s}$ in $t$-distributed measurement errors. Under beta distribution assumptions for the measurement errors, the surface spline plots of EVE of $b_{e}$ and $b_{e s}$ (Fig. 5) are quite similar under small and large samples, but this is not the case for $b_{s}$. The pattern of EMSE under beta distributed measurement errors (Fig. 6) are similar under small and large samples
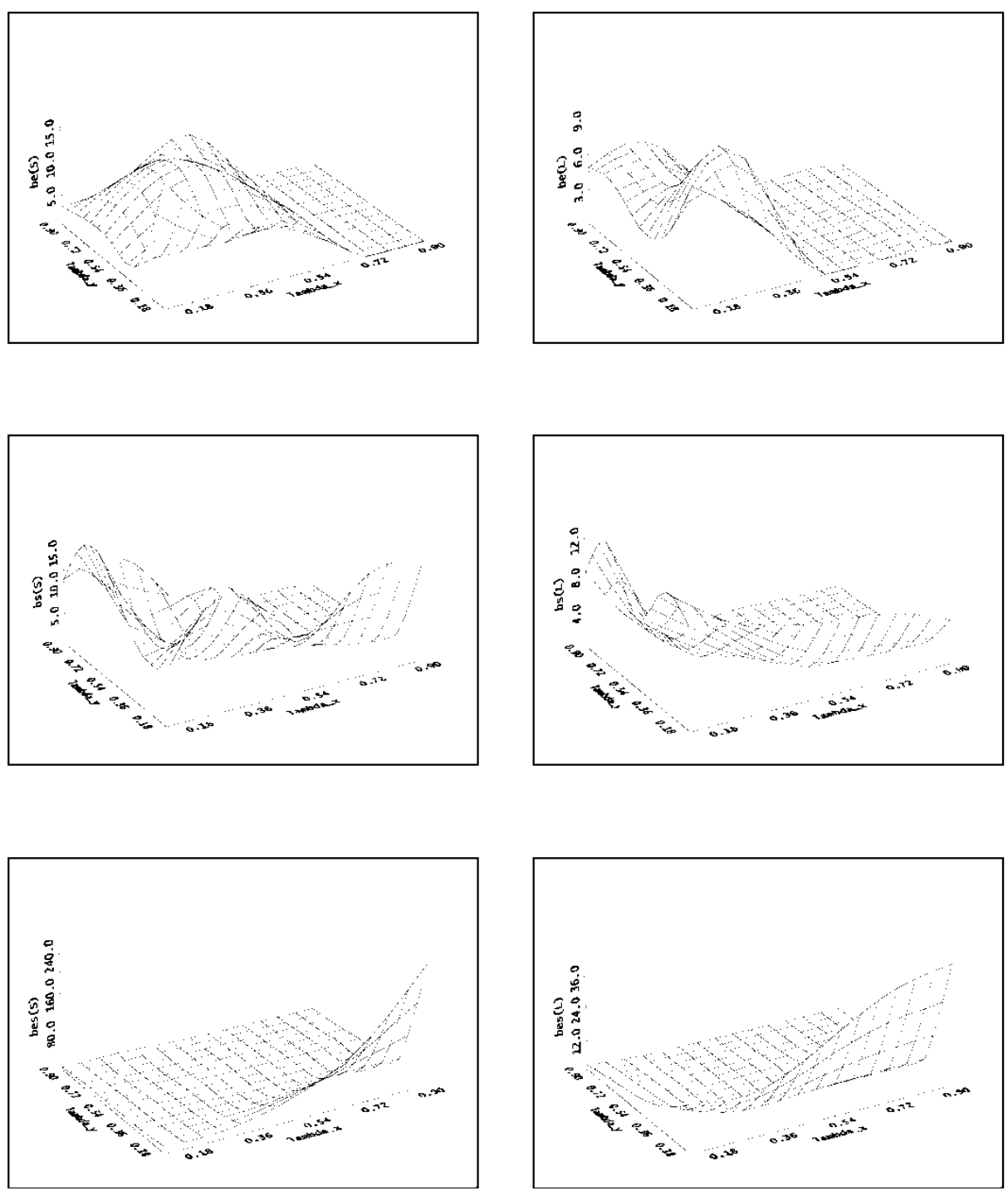

FIGURE 2 EMSE with Normal distribution under small (S) and large (L) samples. The values on vertical axis are the EMSEs of $b_{e}$ (be), $b_{s}(\mathrm{bs})$ and $b_{e s}$ (bes). 

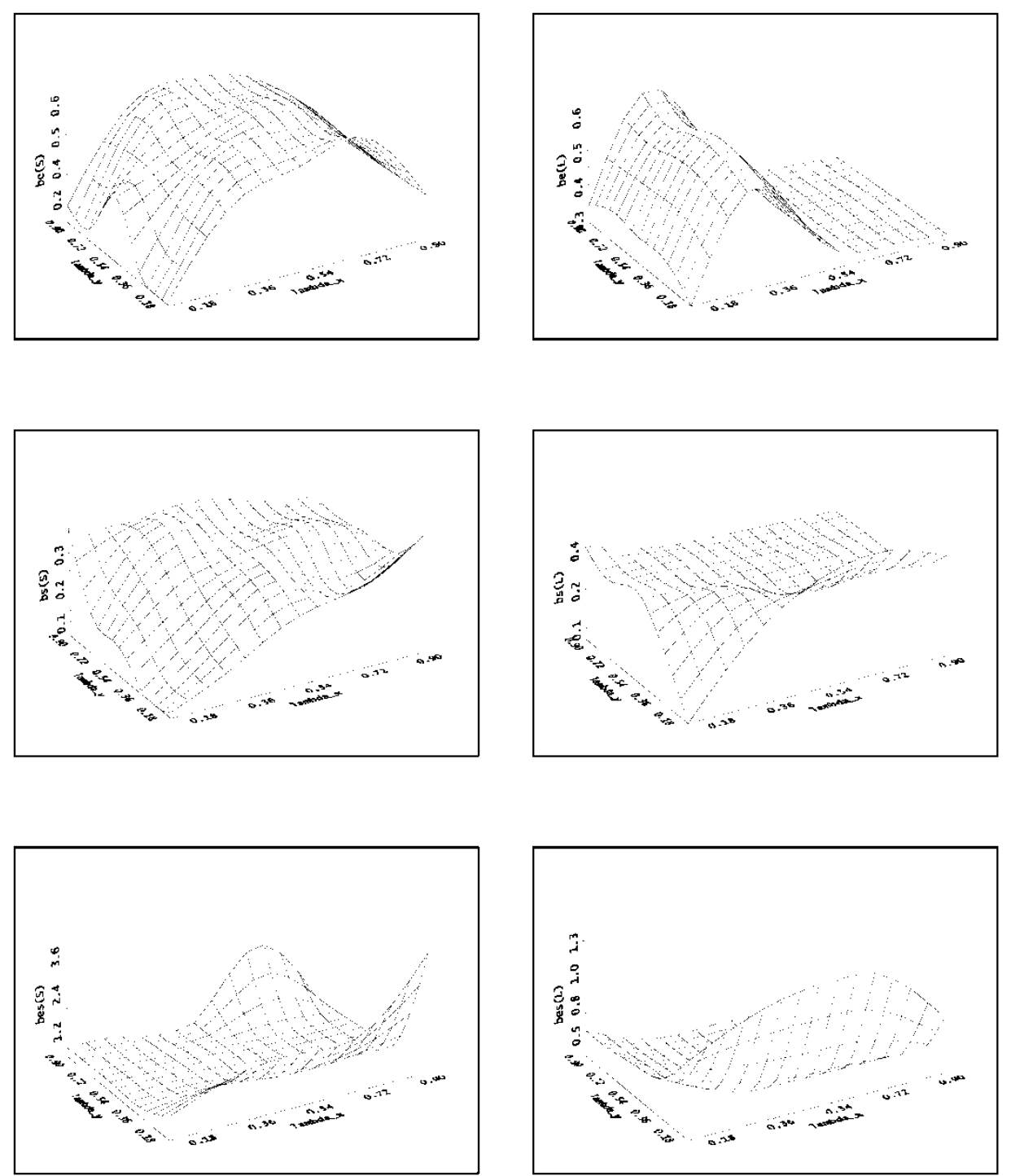

FIGURE 3 EVE with t-distribution under small (S) and large (L) samples. The values on vertical axis are the EVEs of $b_{e}$ (be), $b_{s}$ (bs) and $b_{e s}$ (bes).

for $b_{e s}$, but different for $b_{e}$ and $b_{s}$. The magnitudes of EMSEs (Fig. 6) of $b_{e}$ and $b_{s}$ are smaller in large samples as compared with small samples whereas EMSE of $b_{e s}$ is smaller in small samples. Similar large and small sample patterns can be seen in the EVE (Fig. 7) of all the three estimators under Weibull distributed measurement errors, but the EMSE plots, (Fig. 8) of $b_{e}$ and $b_{s}$ are similar whereas $b_{e s}$ is different. It is generally observed that in the case of similar surface spline plots, the plots for large samples are smoother than those of small samples.

Now we restrict our attention to the inter-comparison of effects of measurement errors under different distributions. Firstly, we compare the $t$-distribution $\left(\gamma_{1}=0, \gamma_{2}=3.154\right)$ (Figs. 3 and 4) with the normal distribution (Figs. 1 and 2) in order to see the effect of skewness of the measurement error distributions on the performance of the estimators. The pattern of the surface spline plots of all three estimators is quite similar with respect to the magnitude 

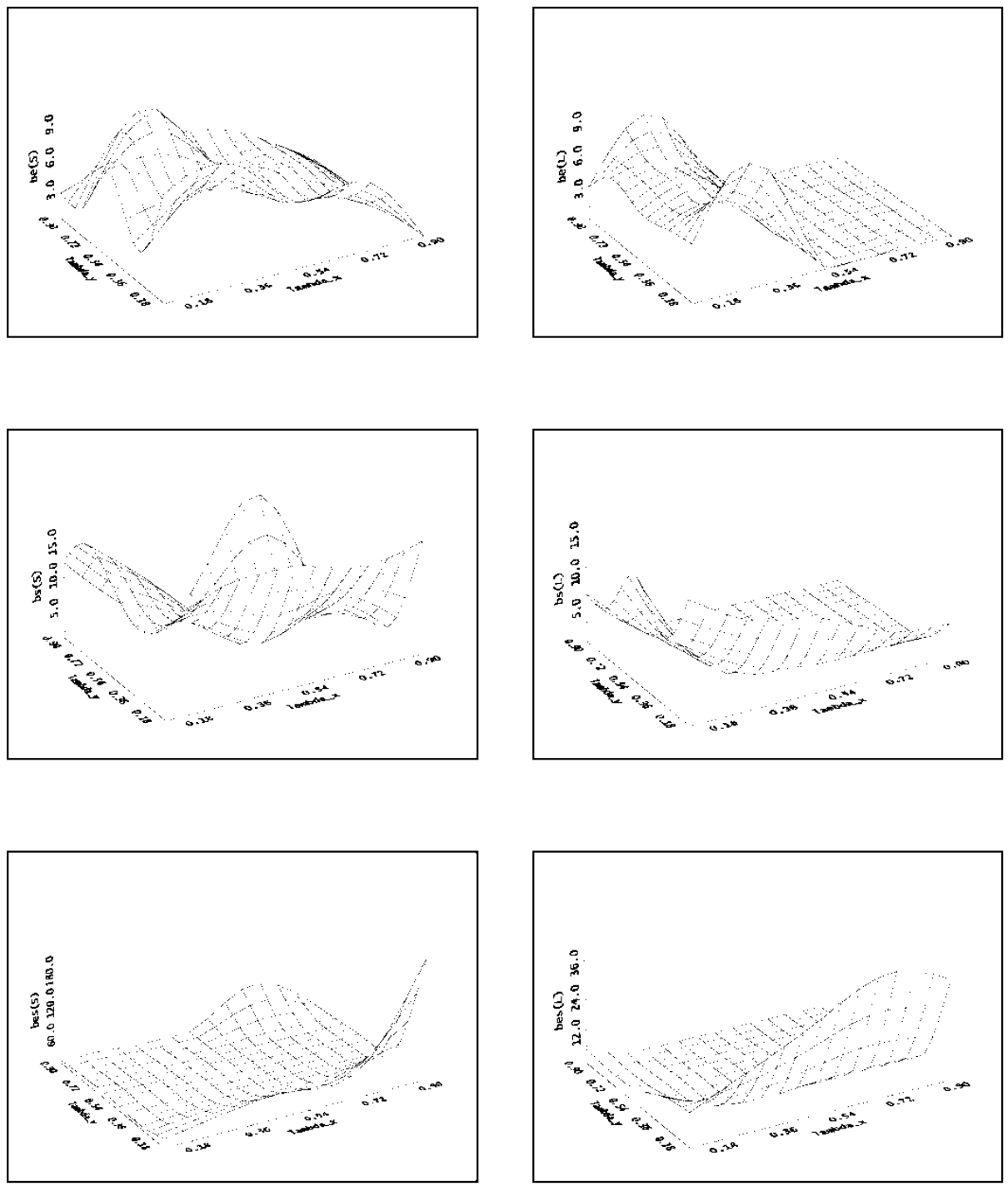

FIGURE 4 EMSE with $t$-distribution under small (S) and large (L) samples. The values on vertical axis are the EMSEs of $b_{e}$ (be), $b_{s}(\mathrm{bs})$ and $b_{e s}$ (bes).

of EVE and their EMSEs. This indicates that skewness of the measurement error distribution does not play a major role, or may have little effect on the performance of the estimators, an assumption also indicated by the expressions for EVE and EMSE under first-order large sample asymptotic approximations.

Next, we compare the performance of beta distributed measurement errors (Figs. 5 and 6) with normally distributed measurement errors (Figs. 1 and 2). We note that our choices of beta distributions have non-zero coefficients of skewness and kurtosis $\left(\gamma_{1}=0.510, \gamma_{2}=3.308\right)$. Here, the effect of skewed and peaked distributions is seen more clearly in small samples $(n=15)$. There is a wave pattern in the surface spline plots in case of EVEs of $b_{e}$ and $b_{s}$, i.e., the bias first increases, then decreases and finally increases again with increasing values of both the reliability ratios. The behavior of EVEs of beta and normally distributed 

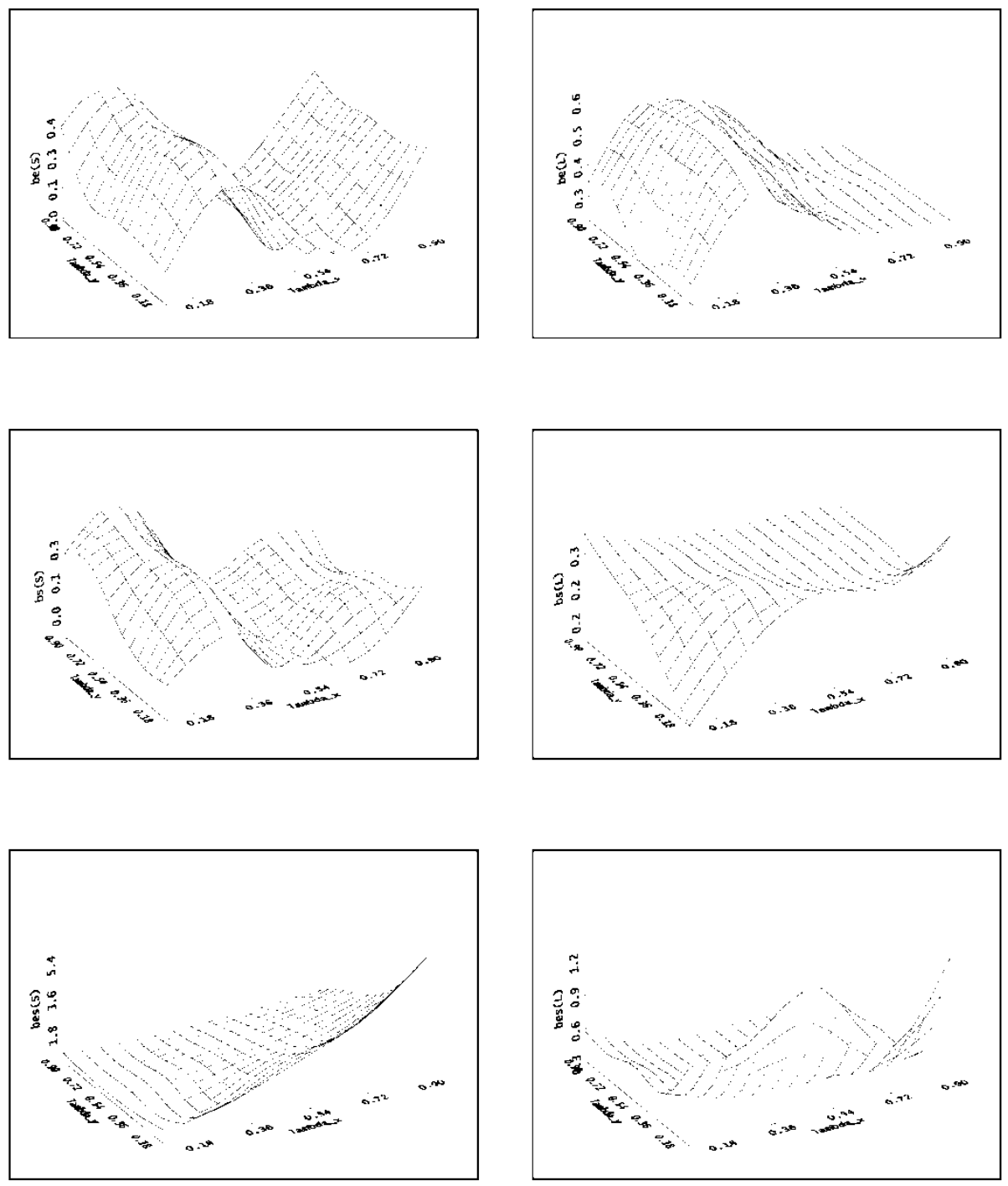

FIGURE 5 EVE with Beta distribution under small (S) and large (L) samples. The values on vertical axis are the EVEs of $b_{e}$ (be), $b_{s}$ (bs) and $b_{e s}$ (bes).

measurement errors is quite similar in large samples $(n=43)$ as compared with small samples $(n=15)$ except in case of EVE of $b_{e s}$, where the behavior is similar under both small and large samples. The direction of bias also changes from positive to negative in small samples whereas the bias is generally positive in large samples. The direction of bias for $b_{e s}$ is generally positive and its magnitude remains stable with little variations over a large range of values of $\lambda_{x}$ and $\lambda_{y}$. In large samples, a wave pattern is observed in the bias of $b_{e}$. While comparing the EMSEs, we observe that the patterns are similar but their magnitudes differ for $b_{e s}$ under both small and large samples. The patterns, as well as the magnitudes, of EMSEs of $b_{e}$ and $b_{s}$ are different under both small and large samples. The higher magnitude, of mean squared errors reflects the effect of non-normality of the distribution of the measurement errors.

Lastly, we take up the Weibull distribution. The Weibull distributed measurement errors are common in life testing experiments. The effect of non-normally distributed measurement 

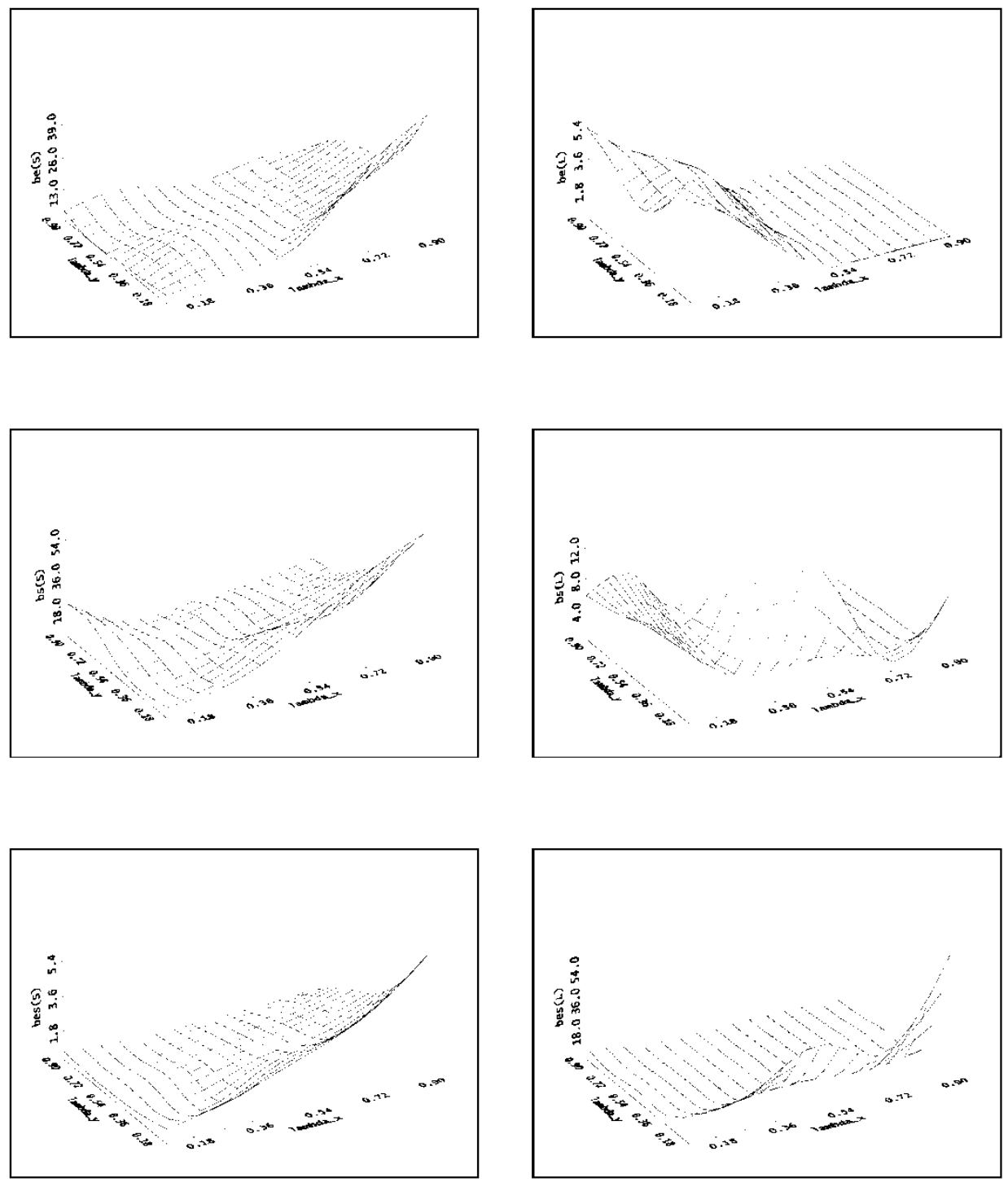

FIGURE 6 EMSE with Beta distribution under small (S) and large (L) samples. The values on vertical axis are the EMSEs of $b_{e}$ (be), $b_{s}(\mathrm{bs})$ and $b_{e s}$ (bes).

errors is most clearly visible in this case. Since the pattern of surface spline plots of EVE and EMSE of all the three estimators under small and large samples are quite similar but irregular, it ensures at least that the behavior revealed by large sample asymptotic approximations is quite satisfactory. But when we compare the plots for the Weibull distribution (Figs. 7 and 8) with those for normal distributions (Figs. 1 and 2), they are quite different. The Weibull distributed measurement errors make the estimators $b_{e}$ and $b_{s}$ highly negatively biased and $b_{e s}$ highly positively biased. They tend to greatly increase the variability of the estimators.

Next, we would like to mention the presence of extremely large values of the simulated estimates. The main reason for the presence of these extremely large values is that as the denominators in the expressions for $b_{e}, b_{s}$ and $b_{e s}$ become very small, the bias and the EMSE can become extremely large. As the sample size $n$ increases, the probability of 

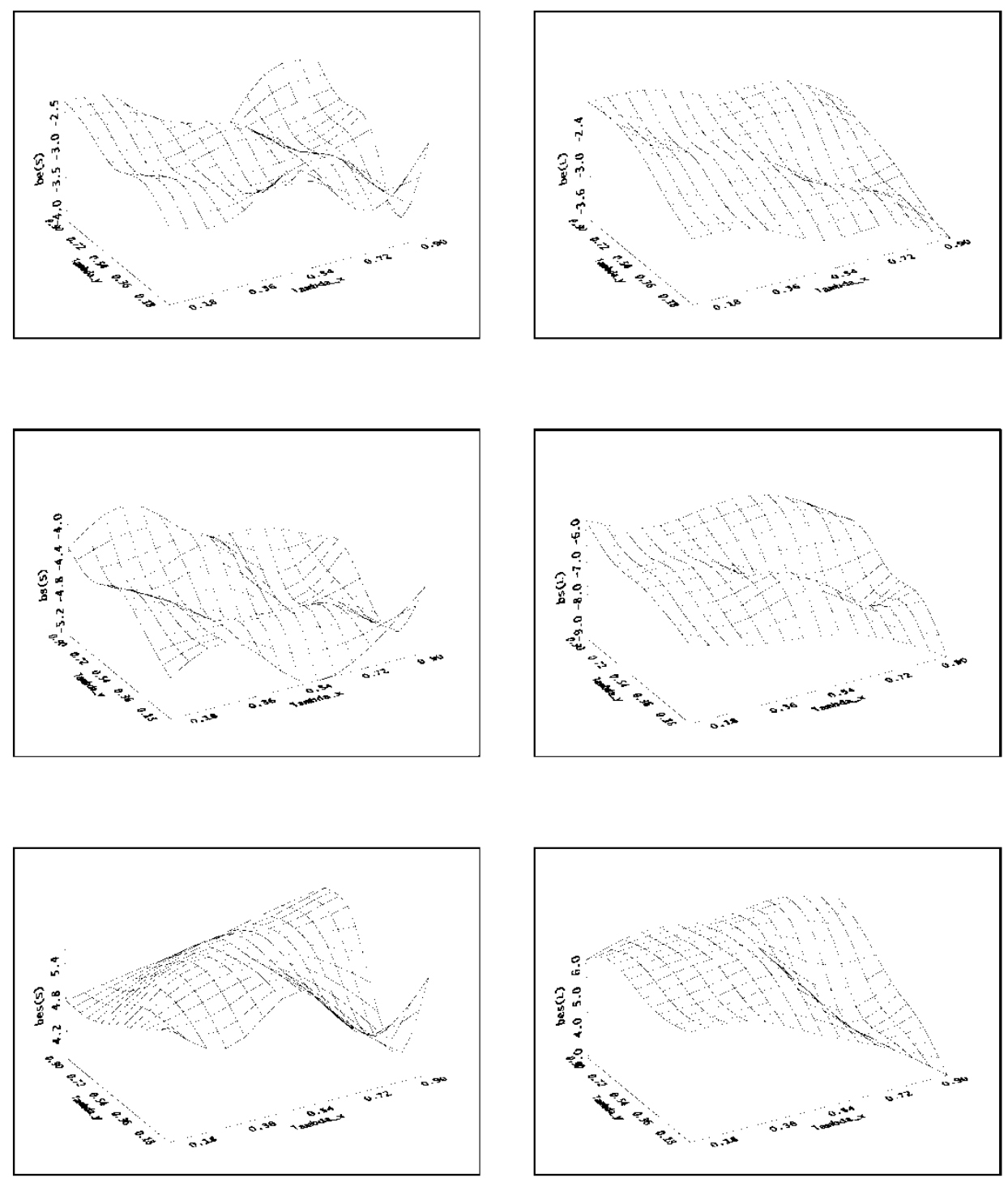

FIGURE 7 EVE with Weibull distribution under small (S) and large (L) samples. The values on vertical axis are the EVEs of $b_{e}$ (be), $b_{s}$ (bs) and $b_{e s}$ (bes).

such extreme values decreases and our asymptotic results therefore do not reflect this problem. For reasons given shortly, we believe that the problem of extreme values of the estimates can be avoided or minimized. Thus in order to get a perhaps more accurate test of the large sample results for the three estimators, we truncated the 5000 values of estimators and their mean squared errors calculated during iterations and recomputed the EVE and EMSE based on values after truncation. We truncated those computed values of the estimators which fell outside the interval $(-100,100)$. (Note that the actual value of the slope parameter being estimated is $\beta=0.3$.) The problem of extreme values affected worst the EVEs and EMSEs in the case of Weibull-distributed measurement errors.

In case the presence of extreme values of the estimates occurs, one can adopt the approach given in Fuller (1987), Srivastava and Shalabh (1997a,b). Their approach essentially involves 

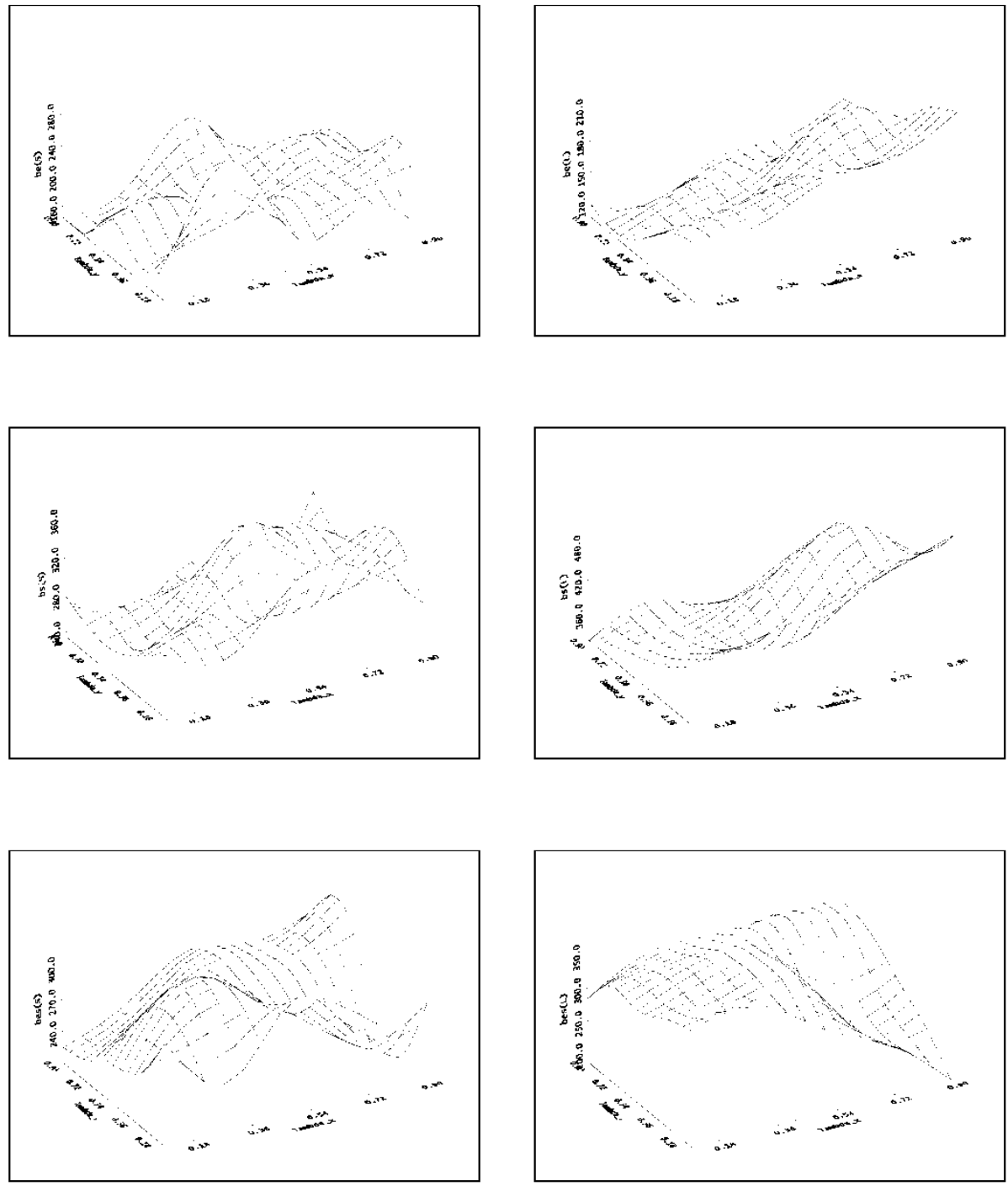

FIGURE 8 EMSE with Weibull distribution under small (S) and large (L) samples. The values on vertical axis are the EMSEs of $b_{e}$ (be), $b_{s}(\mathrm{bs})$ and $b_{e s}$ (bes).

adding some value to the denominator of the estimator. Srivastava and Shalabh (1997a,b) derive a condition to choose the values to be added in the denominator. More detail on this subject would be out of place in the present paper.

Another aspect of the simulation results is to see how close are the results obtained from large sample asymptotic approximations and finite sample simulations. We computed the bias and EMSE of $b_{e}, b_{s}$ and $b_{e s}$ from their large sample asymptotic approximations and compared them with the results obtained from simulation.

We find that for the normal distribution, the large sample asymptotic approximations and simulated results for $b_{e}$ tend to be close as $\lambda_{x}$ and $\lambda_{y}$ increase. For lower values of $\lambda_{x}$ and $\lambda_{y}$, the large sample asymptotic approximations tend to underestimate the true variability. Similar results hold for $b_{s}$ and $b_{e s}$ except for $\lambda_{y}=0.1$ where the large sample asymptotic approximations for the bias are much higher than those obtained by simulation. 
For $t$-distributed measurement errors, the large sample asymptotic approximations and simulated results become closer as $\lambda_{x}$ and $\lambda_{y}$ increase. The EMSE of all three estimators are underestimated at the lower values of $\lambda_{x}$ and $\lambda_{y}$ but they become more accurate for larger values of $\lambda_{x}$ and $\lambda_{y}$.

The bias and mean squared errors under the beta distribution of measurement errors are quite close to each other for all the three estimators except when $\lambda_{x}=0.1$, for which large sample asymptotic approximations overestimate $\beta$ with $b_{e}$ and underestimates with $b_{e s}$. The values of bias and mean squared errors become closer as $\lambda_{x}$ and $\lambda_{y}$ increase. The EMSE of all the three estimators is underestimated by large sample asymptotic approximations for small samples but holds good for large samples. As $\lambda_{x}$ and $\lambda_{y}$ increase, the accuracy of bias and mean squared errors obtained under large sample asymptotic approximations and simulation increases.

It is difficult to comment on the performance of Weibull distributed measurement errors. The results obtained from large sample asymptotic approximations and from simulation for all three estimators differ greatly. Because the effect of outlying values of the estimates is greatly present in the simulation results, better estimates need to be found that reduce this problem before guidelines can be offered.

\section{SOME REMARKS}

We have considered three consistent estimators of the slope parameter $\beta$ arising from knowledge of the exact value of $\sigma_{u}^{2}$ and/or $\sigma_{v}^{2}$, and have analyzed the relative biases and relative mean squared errors of these estimators to order $\mathrm{O}\left(n^{-1}\right)$ when the distributions of the measurement errors are not necessarily normal. Our investigations have revealed that the performance of estimators under non-normal measurement errors may differ considerably from the performance of these estimators when the error distributions are normal. It is interesting to observe that the asymmetry of distributions does not show its influence on the asymptotic properties. However, the kurtosis plays a prominent role. The kurtosis of the error distribution associated with the explanatory variable influences both the bias and mean squared error of the estimator based on the knowledge of $\sigma_{v}^{2}$ while in case of the estimator based on the knowledge of $\sigma_{u}^{2}$, only the mean squared error is influenced by the kurtosis of the error distribution associated with the study variable. When both of the error variances $\sigma_{u}^{2}$ and $\sigma_{v}^{2}$ are utilized in the formulation of the estimator of slope, the bias and mean squared error are influenced by the kurtosis of both error distributions. Besides the kurtosis, the relative biases and the relative mean squared errors of all the three estimators to the given order of approximations are determined by the reliability ratios of both the study and explanatory variables.

It is perhaps no surprise to observe that no estimator dominates the other uniformly with respect to either the criterion of bias or the criterion of mean squared error for all kinds of error distributions. However, we have obtained conditions under which one estimator is superior to the others when the performance criterion is either the relative bias or the relative mean squared error.

When the error distributions are assumed to be mesokurtic or normal, we have compared the estimators with respect to the criterion of the magnitude of bias and have observed that the estimator based on the knowledge of $\sigma_{u}^{2}$ has better performance than the remaining two estimators over a wide range of situations characterized by the values of the reliability ratios $\lambda_{x}$ and $\lambda_{y}$. When the estimators are compared with respect to the criterion of mean squared error, it is interesting to find that the estimator $b_{e s}$ based on the knowledge of both the error 
variances has uniformly superior performance to the estimators which utilize only one of the error variances in their formulation. Perhaps it is worth mentioning that when the errors are normally distributed, Gleser (1985) showed that the estimator $b_{e s}$ is the maximum likelihood estimator and therefore it has the smallest asymptotic variance among all estimators. So far as the performance of estimators using one of the error variances is concerned, it is observed that the estimator based on the knowledge of $\sigma_{v}^{2}$ has better performance than the estimator based on $\sigma_{u}^{2}$ when $\lambda_{x}$ is less than $\lambda_{y}$. This result is reversed when $\lambda_{x}$ is greater than $\lambda_{y}$.

The results obtained by simulation can be combined with the dominance conditions obtained by large sample asymptotic approximations to gain a better view of the performance of estimators. The a priori knowledge about $\lambda_{x}$ and $\lambda_{y}$ will help in deciding which estimator out of the three will perform better under any particular distribution of measurement errors. Thus one may choose the appropriate piece of information in terms of measurement error variances to formulate the estimator.

\section{Acknowledgement}

The first author acknowledges support from Department of Science \& Technology, Govt. of India through BOYSCAST fellowship.

\section{References}

Cheng, C. and Van Ness, J. W. (1991). On the unreplicated ultra-structural model. Biometrika, 78, 442-445.

Cheng, C. L. and Van Ness, J. W. (1994). On estimating linear relationships when both variables are subject to errors. Journal of the Royal Statistical Society, B, 56, 167-183.

Cheng, C. L. and Van Ness, J. W. (1999). Statistical Regression with Measurement Errors. Arnold Publishers.

Fuller, W. A. (1987). Measurement Error Models. John Wiley.

Gleser, L. J. (1985). A note on G.R. Dolby's unreplicated ultrastructural model. Biometrika, 72, 117-124.

Gleser, L. J. (1992) The importance of assessing measurement reliability in multivariate regression. Journal of American Statistical Association, 87, 419, 696-407.

Gleser, L. J. (1993). Estimators of slope in linear errors-in-variables regression models when the predictors have known reliability matrix. Statistics \& Probability Letters, 17(2), 113-121.

Moore, D. S. (2000). The Basic Practice of Statistics. W.H. Freeman, New York.

Patefield, W. M. (1978). The unreplicated ultrastructural relation: Large sample properties. Biometrika, 65, 535-540.

Srivastava, A. K. and Shalabh. (1997a). Consistent estimation for the non-normal ultrastructural model. Statistics and Probability Letters, 34, 67-73.

Srivastava, A. K. and Shalabh. (1997b). Improved estimation of slope parameter in a linear ultrastructural model when measurement errors are not necessarily normal. Journal of Econometrics, 78, 133-157.

Wong, M. Y. (1993). Estimation of the slope in linear non-normal structural relationships. Communications in Statistics - Theory \& Methods, 22, 2421-2428.

\section{APPENDIX}

Following Srivastava and Shalabh (1997a), let us write

$$
\begin{aligned}
u & =\operatorname{Col}\left(u_{1}, u_{2}, \ldots, u_{n}\right), v=\operatorname{Col}\left(v_{1}, v_{2}, \ldots, v_{n}\right) \\
w & =\operatorname{Col}\left(w_{1}, w_{2}, \ldots, w_{n}\right), m=\operatorname{Col}\left(m_{1}, m_{2}, \ldots, m_{n}\right), \\
h & =\frac{1}{n^{1 / 2} \sigma_{v}^{2}}\left[\left(w^{\prime} A w-n \sigma_{w}^{2}\right)+v^{\prime} A(m+w)+2 m^{\prime} A w+\frac{1}{\beta} u^{\prime} A(m+w+v)\right] \\
h^{*} & =\frac{1}{n^{1 / 2} \sigma_{v}^{2}}\left[\frac{1}{\beta^{2}}\left(u^{\prime} A u-n \sigma_{u}^{2}\right)+\frac{1}{\beta} u^{\prime} A(m+w-v)-v^{\prime} A(m+w)\right],
\end{aligned}
$$


where $A$ is a $n \times n$ matrix with all diagonal elements equal to $(n-1) / n$ and all off-diagonal elements equal to $-1 / n$.

Using (2.1)-(2.4), we can express

$$
\begin{aligned}
& s_{x y}=\beta \sigma_{v}^{2}\left(\frac{\lambda_{x}}{1-\lambda_{x}}+\frac{h}{n^{1 / 2}}\right) \\
& s_{y y}=\sigma_{u}^{2}+\beta s_{x y}+\frac{\beta^{2} \sigma_{v}^{2} h^{*}}{n^{1 / 2}}
\end{aligned}
$$

so that

$$
\begin{aligned}
\left(\frac{b_{s}-\beta}{\beta}\right) & =\frac{s_{y y}-\sigma_{u}^{2}-\beta s_{x y}}{\beta s_{x y}} \\
& =\frac{\left(1-\lambda_{x}\right) h^{*}}{n^{1 / 2} \lambda_{x}}\left[1+\frac{\left(1-\lambda_{x}\right) h}{n^{1 / 2} \lambda_{x}}\right]^{-1} .
\end{aligned}
$$

Expanding and retaining terms up to order $\mathrm{O}\left(n^{-1}\right)$, we get

$$
\left(\frac{b_{s}-\beta}{\beta}\right)=\frac{\left(1-\lambda_{x}\right) h^{*}}{n^{1 / 2} \lambda_{x}}-\frac{\left(1-\lambda_{x}\right)^{2} h h^{*}}{n \lambda_{x}^{2}}
$$

whence, up to order $\mathrm{O}\left(n^{-1}\right)$ only, we have

$$
\begin{aligned}
E\left(\frac{b_{s}-\beta}{\beta}\right) & =\frac{\left(1-\lambda_{x}\right)}{n^{1 / 2} \lambda_{x}} E\left(h^{*}\right)-\frac{\left(1-\lambda_{x}\right)^{2}}{n \lambda_{x}^{2}} E\left(h h^{*}\right) \\
E\left(\frac{b_{s}-\beta}{\beta}\right)^{2} & =\frac{\left(1-\lambda_{x}\right)^{2}}{n \lambda_{x}^{2}} E\left(h^{*^{2}}\right) .
\end{aligned}
$$

If $Z$ denotes a $n \times 1$ random vector such that its elements are independently and identically distributed with mean 0 , variance 1 and kurtosis coefficient $\gamma_{2}$, and $B$ denotes any nonstochastic matrix, we have

$$
\begin{aligned}
E\left(Z^{\prime} B Z\right) & =(\operatorname{tr} B) \\
E\left(Z^{\prime} A Z \cdot Z^{\prime} B Z\right) & =\left(1+\frac{\gamma_{2}}{n}\right)(n-1)(\operatorname{tr} B)+2(\operatorname{tr} A B) .
\end{aligned}
$$

Using these results along with the properties of $u, v$ and $w$, it is easy to see that

$$
\begin{aligned}
E\left(h^{*}\right) & =-\frac{q}{n^{1 / 2}}+\mathrm{O}\left(n^{-1}\right) \\
E\left(h h^{*}\right) & =-\left[\frac{\lambda_{x}+q\left(1-2 \lambda_{x}\right)}{1-\lambda_{x}}\right]+\mathrm{O}\left(n^{-1}\right) \\
E\left(h^{*^{2}}\right) & =\left(\frac{q+\lambda_{x}}{1-\lambda_{x}}\right)+q^{2}\left(2+\gamma_{2 u}\right)+\mathrm{O}\left(n^{-1}\right) .
\end{aligned}
$$

Substituting these results, we find the expressions (3.2) of Theorem 1 and (3.16) of Theorem 2.

For the derivation of similar results for the estimator $b_{e s}$, we write

$$
s_{x x}=\sigma_{v}^{2}\left[\frac{1}{1-\lambda_{x}}+\frac{d}{n^{1 / 2}}\right],
$$


where

$$
d=\frac{1}{n^{1 / 2} \sigma_{v}^{2}}\left[\left(v^{\prime} A v-n \sigma_{v}^{2}\right)+\left(w^{\prime} A w-n \sigma_{w}^{2}\right)+2 m^{\prime} A(v+w)+2 v^{\prime} A w\right]
$$

Now we can express

$$
\begin{aligned}
t & =\frac{1}{2 s_{x y}}\left[s_{y y}-\frac{\sigma_{u}^{2}}{\sigma_{v}^{2}} s_{x x}\right] \\
& =\frac{\beta}{2}\left[(1-q)+\frac{\left(1-\lambda_{x}\right)\left(h+h^{*}-q d\right)}{n^{1 / 2} \lambda_{x}}\right]\left[1+\frac{\left(1-\lambda_{x}\right) h}{n^{1 / 2} \lambda_{x}}\right]^{-1} \\
& =\frac{\beta}{2}\left[(1-q)+\frac{k}{n^{1 / 2}}-\frac{k k^{*}}{n}\right]+\mathrm{O}_{p}\left(n^{3 / 2}\right)
\end{aligned}
$$

where

$$
\begin{aligned}
k= & \left(\frac{1-\lambda_{x}}{\lambda_{x}}\right)\left[h^{*}+q(h-d)\right] \\
= & \left(\frac{1-\lambda_{x}}{n^{1 / 2} \lambda_{x} \sigma_{v}^{2}}\right)\left[\frac{1}{\beta^{2}}\left(u^{\prime} A u-\sigma_{u}^{2}\right)+\left(\frac{1+q}{\beta}\right) u^{\prime} A(m+w)-\left(\frac{1-q}{\beta}\right) u^{\prime} A v\right. \\
& \left.-q\left(v^{\prime} A v-n \sigma_{v}^{2}\right)-(1+q) v^{\prime} A(m+w)\right] \\
= & \left(\frac{1-\lambda_{x}}{\lambda_{x}}\right) h .
\end{aligned}
$$

Similarly, we have

$$
\begin{aligned}
\left(\frac{t^{2}}{\beta^{2}}+q\right)^{1 / 2}= & \frac{1}{2}\left[(1-q)^{2}+4 q+\frac{2(1-q) k}{n^{1 / 2}}+\frac{k^{2}-2(1-q) k k^{*}}{n}+\mathrm{O}_{p}\left(n^{-3 / 2}\right)\right]^{1 / 2} \\
= & \left(\frac{1+q}{2}\right)\left[1+\frac{2(1-q) k}{n^{1 / 2}(1+q)^{2}}+\frac{k^{2}-2(1-q) k k^{*}}{n}+\mathrm{O}_{p}\left(n^{-3 / 2}\right)\right]^{1 / 2} \\
= & \left(\frac{1+q}{2}\right)\left[1+\frac{(1-q) k}{n^{1 / 2}(1+q)^{2}}+\frac{1}{n(1+q)^{2}}\left\{\frac{2 q k^{2}}{(1+q)^{2}}-(1-q) k k^{*}\right\}\right] \\
& +\mathrm{O}_{p}\left(n^{-3 / 2}\right) .
\end{aligned}
$$

Thus we find

$$
\begin{aligned}
\left(\frac{b_{e s}-\beta}{\beta}\right) & =\left(\frac{t-\beta}{\beta}\right)+\left(\frac{t^{2}}{\beta^{2}}+q\right)^{1 / 2} \\
& =\frac{k}{n^{1 / 2}(1+q)}+\frac{1}{n(1+q)}\left[\frac{q k^{2}}{(1+q)^{2}}-k k^{*}\right]+\mathrm{O}_{p}\left(n^{-3 / 2}\right)
\end{aligned}
$$


whence the relative bias and relative mean squared error of the estimator $b_{e s}$ to order $\mathrm{O}\left(n^{-1}\right)$ is

$$
\begin{aligned}
E\left(\frac{b_{e s}-\beta}{\beta}\right) & =-\frac{1}{n^{1 / 2}(1+q)} E(k)+\frac{1}{n(1+k)}\left[\frac{q}{(1+q)^{2}} E\left(k^{2}\right)+E\left(k k^{*}\right)\right] \\
E\left(\frac{b_{e s}-\beta}{\beta}\right)^{2} & =\frac{1}{n(1+q)^{2}} E\left(k^{2}\right) .
\end{aligned}
$$

Observing that

$$
\begin{aligned}
E(k) & =0 \\
E\left(k^{2}\right) & =\left(\frac{1-\lambda_{x}}{\lambda_{x}}\right)^{2}\left[q(1+q)^{2}+\left(\frac{\lambda_{x}}{1-\lambda_{x}}\right)(1+q)^{3}+q^{2}\left(\gamma_{2 u}+\gamma_{2 v}\right)\right]+\mathrm{O}\left(n^{-1}\right) \\
E\left(k k^{*}\right) & =\left(\frac{1-\lambda_{x}}{\lambda_{x}}\right)^{2}(q-1)\left[q+(1+q)\left(\frac{\lambda_{x}}{1-\lambda_{x}}\right)\right]+\mathrm{O}\left(n^{-1}\right)
\end{aligned}
$$

and substituting these expressions, we obtain the results (3.3) and (3.17) stated in Theorems 1 and 2 respectively. 